\title{
Characterization of Biologically Available Wood Combustion Particles in Cell Culture Medium
}

\author{
Susanne Gauggel ${ }^{1}$, Cassandra Derreza-Greeven ${ }^{1,3}$, Julia Wimmer ${ }^{1,4}$, \\ Mark Wingfield ${ }^{2}$, Bart van der Burg ${ }^{5}$, and Daniel R. Dietrich ${ }^{1}$ \\ ${ }^{1}$ Human and Environmental Toxicology, University of Konstanz, Konstanz, Germany; ${ }^{2}$ Malvern Instruments GmbH, \\ Herrenberg, Germany; ${ }^{3}$ Current address: Institut für Energie und Umweltforschung (IFEU), Heidelberg, Germany; \\ ${ }^{4}$ Current address: Kompetenzzentrum Obstbau-Bodensee, Ravensburg-Bavendorf, Germany; ${ }^{5}$ BioDetection Systems b.v., \\ Amsterdam, The Netherlands
}

\begin{abstract}
Summary
Combustion of wood produces particulate matter (PM) emissions having the potential to induce respiratory tract diseases in humans. To date, however, few, if any, in vitro submerse exposure adverse effect studies characterized the actual particle characteristics within the culture medium. Indeed, the availability of particles and adsorbed toxic compounds in liquids may depend on particle characteristics, i.e. aggregation, size, composition, type (complex solids, salts, etc.) and thus affect toxicity. Using polystyrene nanoparticles as reference, the particle size distribution and aggregation status of wood furnace PM and quartz particles in standard cell culture medium and water was characterized. Characterization was carried out via scanning electron microscopy (SEM), light microscopy, dynamic light scattering (DLS), and laser diffraction. Moreover, the biological availability of particles and adsorbed polycyclic aromatic hydrocarbons was tested using an Ah-receptor reporter gene assay, which demonstrated that particle characterization and knowledge of toxin bioavailability prior to experimentation is key for understanding potential biological interactions.
\end{abstract}

Keywords: particulate matter, wood combustion, particle characterization, polycyclic aromatic hydrocarbons $(P A H)$, bioavailability

\section{Introduction}

Although humans have always been exposed to particulate matter (PM) emerging from natural sources, industrialization-associated PM (traffic, combustion sources, power plants, etc.) has continuously increased. The latter represents a risk for human health (Pope et al., 2002), as anthropogenic PM has longer atmospheric residential times and may contain toxic compounds. The recent focus on PM from traffic, industry, and combustion processes, and the corresponding adverse health effects (Billet et al., 2008; Danielsen et al., 2011; Gualtieri et al., 2008; Pope and Dockery, 2006) have resulted in a mandatory reduction of traffic exhausts and of PM in general within the European Community (European Union, 2008).

In contrast, potential adverse health effects of PM originating from the combustion of wood are less well characterized (Naeher et al., 2005). However, increased pricing and perceived shortage of primary energy sources resulted in an increasing demand for affordable renewable energy sources, e.g., wood. Moreover, carbon-based renewable fuel is regarded as $\mathrm{CO}_{2}$ neu- tral, hence politically supported and considered important for climate protection (Quaak et al., 1999), resulting in increased use of wood burners for residential heating. The latter contributes to high local particulate matter emissions (Naeher et al., 2007) which have led to restrictions of wood burners in smogprone cities, e.g., Christchurch, New Zealand. ${ }^{1}$ Wood burners allowed in New Zealand have a maximum emission of $1.5 \mathrm{~g}$ PM per kilogram of dry wood burned and a thermal efficiency of $\geq 65 \%$. However, reality shows that once a wood burner is installed, woods with differing levels of dryness are burnt, resulting in incomplete combustion and PM emissions that differ dramatically with regard to particle size and toxin composition.

As the latter parameters govern the potential adverse effects of PM in humans once inhaled, testing of wood combustion PM for health effects assessment largely relies on in vitro suspension experiments with particles collected on filters in smoke tubes or obtained via electrostatic precipitators and roughly characterized in the air flow with special devices, e.g., mobile particle size scanners. However, to investigate their toxic potential via in vitro studies, cell cultures are challenged

Received October 26, 2011; accepted in revised form March 19, 2012.

1 http://www.mfe.govt.nz/laws/standards/woodburners/authorised-woodburners.html 
with PM samples via submerse exposure or exposure at the air-liquid-interface. Similar to the in vivo situation, where PM contacts the epithelial lining fluid, PM in submerse cultures or at the air-liquid-interface interact with the cell culture medium. Upon suspension in liquids the physico-chemical characteristics of PM may change, due to aggregation, wetting or dissolution, with ensuing consequences for the bioavailability of particulates and/or adsorbed toxins and thus for the type and intensity of the effects observed. Hence, toxicological studies of PM must always be accompanied by a characterization of particles in liquids.

Indeed, particle aggregation or dissociation is influenced by physico-chemical characteristics of the particles themselves, as well as by characteristics of the suspension media (Teeguarden et al., 2007). Particle properties governing aggregation and dissociation, and thus the bioavailability of PM in the in vitro cell system are morphology, size, mass, shape, surface area, surface coating, charge, and composition, including hazardous compounds, e.g., polycyclic aromatic hydrocarbons and heavy metals. Conversely, the characteristics of the liquids used for the cell culture system, e.g., viscosity, $\mathrm{pH}$, protein content, and other constituents will influence PM behavior and availability.

In contrast to in vitro toxicology of nanoparticles where an adequate particle characterization is a prerequisite for meaningful outcomes of nanotoxicity studies (Warheit, 2008), the situation with PM is much more complex. Indeed, contrary to the homogeneous synthetically produced nanoparticles, PM is much less homogeneous in size and composition. Thus aggregation of PM and size distribution will affect the outcome of toxicity tests dramatically, as larger particles may settle faster in cell culture and are less available to cells than smaller ones remaining in suspension longer and thus being available for incorporation via endocytosis (Teeguarden et al., 2007).

Obviously there are numerous methods for characterization of nanomaterials (Jones and Grainger, 2009), but no single method will permit a description of PM characteristics sufficient to support an improved interpretation of the in vitro effect data. Consequently, the aim of this study was to assemble a combination of methods (light microscopy, scanning electron microscopy, laser diffraction, dynamic light scattering) assumed to be appropriate for estimation and confirmation of particle properties. This combination of methods was then tested across a variety of particle sample types, including synthetic particles with a given size, as well as PM generated from wood combustion furnaces. Moreover, differences in media were tested by employing water and cell media as particle mixing media, although our expectation was that no overt differences would be observable between particles suspended in ultrapure water and cell culture media. In addition, incompletely and completely combusted PM samples from wood furnaces were compared with regard to particle characterization, salt, elementary carbon, organic carbon, and PAH content as well as to potential differences in toxic effects. The latter allowed us to test the hypothesis that PM from incomplete combustion would be more toxic than PM from complete combustion.

\section{Material and methods}

\section{Chemicals}

Cell culture medium RPMI 1640 with L-Glutamine (Cat. No. E15-840), MEM Alpha modification (Cat. No. E15-832), DMEM/Ham's F12 (Cat. No. E-15813), penicillin/streptomycin (P/S) 100x (Cat. No. P11-010), 1 M HEPES (Cat. No. S11-001) and foetal bovine serum (FBS) (Cat. No. A15-151) were purchased from PAA (Pasching). RPMI cell culture medium for particle characterization was supplemented with $25 \mathrm{mM}$ HEPES, 10\% FBS and 1x P/S. RPMI cell culture medium with $10 \% \mathrm{FBS}$ and $1 \mathrm{x} \mathrm{P} / \mathrm{S}$, referred to as cell culture medium (-HEPES) was employed to control for potential interferences of HEPES in the particle characterization with selected representative samples. Steady-Glo ${ }^{\circledR}$ (Cat. No. E2520) was purchased from Promega. MTT (3-(4,5-dimethylthiazol-2-yl)2,5-diphenyltetrazolium bromide) (Cat. No. M5655) was purchased from Sigma Aldrich.

\section{Reference particles}

Fluorescent and non-fluorescent labeled polystyrene particles of three different sizes each were purchased from Polysciences (17149-10, 17151-10, 17154-10, 08691-10, 07304-15, and 07310-15). Aerosil200 (Cat. No. 112 945-52-5) was purchased from Evonik industries and crystalline quartz DQ25 was obtained from Dörentruper Quarz. The corresponding tables (Tab. $1,2,3)$ list the different particles and their respective sizes, as well as other characteristics according to the manufacturers' specifications.

\section{PM sampling}

Sampling of PM\#01 was carried out in a test station of the German Biomass Research Centre in Leipzig over a period of three days and thus represents a sample from an incomplete combustion: a common wood stove ( $9.0 \mathrm{~kW}$ nominal output), was fired with commercially available non-pretreated standard beech split logs (1.3 kg, specific humidity: $8.9 \%$ ) via hand loading every 30 min. PM was collected in the ash pan of a standard electrostatic precipitator connected to the smoke tube 3-4 m away from the furnace. PM\#02 was obtained from a field experiment in a private household (incomplete combustion) during the 2008/2009

Tab. 1: Overview of polystyrene particles and their respective sizes

\begin{tabular}{|l|l|r|}
\hline Particle type & Description & $\begin{array}{l}\text { Size } \\
\text { according to } \\
\text { manufacturer }\end{array}$ \\
\hline Polystyrene, fluorescent & Fluoresbrite YG & $0.05 \mu \mathrm{m}$ \\
\hline Polystyrene, fluorescent & Fluoresbrite YG & $0.2 \mu \mathrm{m}$ \\
\hline Polystyrene, fluorescent & Fluoresbrite YG & $1 \mu \mathrm{m}$ \\
\hline Polystyrene & Polybead & $0.05 \mu \mathrm{m}$ \\
\hline Polystyrene & Polybead & $0.2 \mu \mathrm{m}$ \\
\hline Polystyrene & Polybead & $1 \mu \mathrm{m}$ \\
\hline
\end{tabular}


heating period. A tile stove $(9.0 \mathrm{~kW}$ nominal output) with an ancillary heating unit was fired with non-pretreated wood briquettes, a mixture of hard and soft wood. An electrostatic precipitator was installed in the smoke tube $(\varnothing 180 \mathrm{~mm})$ between the combustion chamber and the ancillary heating unit to collect the PM sample. The associated smoke tube length after the filter was $3 \mathrm{~m}$ after the ancillary heating unit. PM\#05 was obtained from a wood combustion experiment with an automatic pellet boiler (15 kW nominal output) at the German Biomass Research Centre using "DIN plus" wood pellets. The latter was carried out under optimal combustion conditions and thus represents a complete combustion. The PM\#05 sample was collected in the ash pan of a standard electrostatic precipitator connected to the smoke tube 3-4 m away from the furnace of the automatic pellet boiler.

Tab. 2: Overview of Aerosil200 and its characteristics according to the manufacturer's data sheet

\begin{tabular}{|l|c|c|}
\hline Characteristics & Unit & $\begin{array}{c}\text { Guidance } \\
\text { value }\end{array}$ \\
\hline Specific surface (BET) & $\mathrm{m}^{2} / \mathrm{g}$ & $200 \pm 25$ \\
\hline Average size of primary particles & $\mathrm{nm}$ & 12 \\
\hline $\begin{array}{l}\text { Tamped density } \\
\text { (ex works, variations possible) }\end{array}$ & $\mathrm{g} / \mathrm{l}$ & 50 \\
\hline $\begin{array}{l}\text { Loss on drying, } 2 \mathrm{~h} \text { at } 105^{\circ} \mathrm{C} \\
\text { (ex works, variations possible) }\end{array}$ & weight $\%$ & $\leq 1.5$ \\
\hline $\begin{array}{l}\text { Loss on ignition, } 2 \mathrm{~h} \text { at } 1000^{\circ} \mathrm{C}, \\
\text { based on dry substance }\end{array}$ & weight $\%$ & $\leq 1$ \\
\hline pH value, 4\% dispersion & weight \% & $\geq 99.8$ \\
\hline $\begin{array}{l}\mathrm{SiO} 2 \text { content, based on ignited } \\
\text { substance }\end{array}$ & & $3.7-4.7$ \\
\hline
\end{tabular}

\section{Particle characterization}

Several methods were employed for particle characterization. The methodology is described below, while the advantages and limitations of each method are listed for each particle type in Table 4.

\section{Light microscopy}

PM and quartz samples were diluted to a final concentration of $1 \mathrm{mg} / \mathrm{ml}$ in cell culture medium and ultrapure water. Images were taken with a Nikon Eclipse TS100 microscope equipped with a Nikon DS Camera Head DS-5M.

Scanning electron microscopy (SEM)

For electron microscopy, PM and quartz samples were dry scattered onto stubs and a conductive coating of gold palladium

Tab. 3: Overview of DQ25 and its characteristics according to the manufacturer's data sheet

\begin{tabular}{|l|c|c|}
\hline Characteristics & Unit & $\begin{array}{c}\text { Guidance } \\
\text { value }\end{array}$ \\
\hline Upper grain size $\mathrm{d}_{97 \%}$ & $\mu \mathrm{m}$ & 18 \\
\hline Mean grain size $\mathrm{d}_{50 \%}$ & $\mu \mathrm{m}$ & 4 \\
\hline Bulk density DIN 53468 & $\mathrm{g} / \mathrm{cm}^{3}$ & 0.61 \\
\hline Density (DIN 53193) & $\mathrm{g} / \mathrm{cm}^{3}$ & 2.65 \\
\hline $\mathrm{pH}$-value (DIN 1984) & & 7.0 \\
\hline $\mathrm{SiO}_{2}$ & $\%$ & 99.50 \\
\hline $\mathrm{Al}_{2} \mathrm{O}_{3}$ & $\%$ & 0.20 \\
\hline $\mathrm{Fe}_{2} \mathrm{O}_{3}$ & $\%$ & 0.03 \\
\hline $\mathrm{CaO}+\mathrm{MgO}$ & $\%$ & $<0.04$ \\
\hline $\mathrm{Na}_{2} \mathrm{O}+\mathrm{K}_{2} \mathrm{O}$ & $\%$ & $<0.04$ \\
\hline $\begin{array}{l}\mathrm{Loss} \text { on ignition } 1000^{\circ} \mathrm{C} \\
(\mathrm{DIN} \mathrm{EN} \mathrm{ISO} \mathrm{3262-7)}\end{array}$ & $\%$ & $<0.20$ \\
\hline
\end{tabular}

Tab. 4: Overview of applied measurement methods and their suitability for the different particle samples

(PS, polystyrene; n.m.: no measurement; ++: optimal; +: acceptable; \pm : suboptimal; -: inadequate)

\begin{tabular}{|c|c|c|c|c|c|c|c|c|}
\hline Methods Sample Types & $\begin{array}{l}\text { PS, } \\
1,000 \mathrm{~nm}\end{array}$ & $\begin{array}{l}\text { PS, } \\
200 \mathrm{~nm}\end{array}$ & $\begin{array}{l}\text { PS, } \\
50 \mathrm{~nm}\end{array}$ & DQ25 & $\begin{array}{l}\text { Aerosil } \\
200\end{array}$ & PM\#01 & PM\#02 & PM\#05 \\
\hline Light microscopy & ++ & ++ & - & + & + & + & + & n.m. \\
\hline SEM 505, Phillips & ++ & + & - & + & + & + & + & + \\
\hline SEM S-4700, Hitachi & n.m. & n.m. & \pm & n.m. & n.m. & n.m. & n.m. & n.m. \\
\hline Zetasizer (ZetaPot.) 5 nm-10 $\mu \mathrm{m}$ & ++ & ++ & ++ & + & + & + & + & + \\
\hline Zetasizer (DLS) $0.6 \mathrm{~nm}-6 \mu \mathrm{m}$ & ++ & ++ & ++ & + & + & + & + & + \\
\hline Mastersizer $20 \mathrm{~nm}-2,000 \mu \mathrm{m}$ & ++ & ++ & \pm & ++ & ++ & ++ & ++ & ++ \\
\hline Nanosight $10 \mathrm{~nm}-1,000 \mathrm{~nm}$ & \pm & ++ & ++ & \pm & \pm & \pm & \pm & n.m. \\
\hline
\end{tabular}


(5 nm) was applied. Polystyrene particles were diluted with ethanol and a subsample dropped onto stubs and air dried before a conductive coating of gold palladium $(5 \mathrm{~nm})$ was applied. SEM was performed with a Phillips (FEI) SEM 505. Because of limited resolution of the SEM 505 a Hitachi Field-Emission SEM S-4700 was used for the smallest polystyrene particles (50 $\mathrm{nm})$.

\section{Sample preparation for Zetasizer, Mastersizer and Nanosight measurements}

All PM suspensions were freshly prepared in ultrapure water and cell culture medium prior to measurement. Polystyrene particle suspensions of $1 \mathrm{mg} / \mathrm{ml}, 50 \mu \mathrm{g} / \mathrm{ml}, 0.525 \mathrm{mg} / \mathrm{ml}, 2.5 \mu \mathrm{g} / \mathrm{ml}$ for measurements with the Zetasizer (zeta potential), Zetasizer (DLS), Mastersizer (laser diffraction) and Nanosight were prepared, respectively. Prior to measurements, polystyrene suspensions were ultrasonicated in a volume of $2 \mathrm{ml}$ with a frequency of $35 \mathrm{kHz}$ for $5 \mathrm{~min}$ (Elma T470/H, 50/60 Hz) in $2 \mathrm{ml}$ microcentrifuge tubes. Quartz and PM sample suspensions for measurements contained $0.5,1$, and $2 \mathrm{mg} / \mathrm{ml}$, whereas suspensions used for centrifugation experiments contained $1 \mathrm{mg} / \mathrm{ml}$ of PM or quartz. Because of the limited sample amount only $1 \mathrm{mg} / \mathrm{ml}$ suspensions were prepared for PM\#05. PM suspensions were centrifuged (Heraeus Megafuge 1.0/ HeraeusMegafuge 1.0 R) for $5 \mathrm{~min}$ at 1,000, 2,000 and 3,000 $\mathrm{x} \mathrm{g}$, quartz suspensions at $111 \mathrm{x}$ g. Supernatants were collected and the resulting pellets resuspended in $10 \mathrm{ml}$ cell culture medium or water. As sonication may dramatically influence the suspension characteristics (Taurozzi et al., 2010), all available details for suspension sonication are described below. All PM and quartz suspensions were ultrasonicated with an ultrasonic frequency of $35 \mathrm{kHz}$ (Elma $\mathrm{T} 470 / \mathrm{H}, 50 / 60 \mathrm{~Hz}$ ) for $15 \mathrm{~min}$ in $50 \mathrm{ml}$ centrifuge tubes (conical bottom) with volumes of 5-10 ml. A higher volume was required for Mastersizer measurements of polystyrene, quartz and PM particles. For these $50 \mathrm{ml}$ centrifuge tubes (conical bottom) were chosen and filled with volumes of 20-50 $\mathrm{ml}$ (depending on particle type). The time delay between ultrasonication and actual measurement of the samples was $1.5 \mathrm{~h}, 0.5 \mathrm{~h}$, and $1.5 \mathrm{~h}$ for the Nanosight, Zetasizer, and Mastersizer, respectively.

\section{Zeta potential (Zetasizer) and $\mathrm{pH}$}

The zeta potential gives an indication of the potential stability (i.e., likelihood of aggregation) of a suspension. In general, a potential between $+30 \mathrm{mV}$ and $-30 \mathrm{mV}$ is regarded as instable (aggregated suspensions), whereas values lower than $-30 \mathrm{mV}$ or higher than $+30 \mathrm{mV}$ represent "homogenous" suspensions ${ }^{2}$. A Zetasizer Nano ZS (Malvern Instruments GmbH, Herrenberg, Germany) was used to measure the zeta potential at $25^{\circ} \mathrm{C}$ in zeta cells in three measurements with 30 cycles each. As the medium measurements with the standard zeta cells with gold electrodes appeared problematic due to potential interactions between the electrodes and the media, most likely induced by its high ionic strength, control measurements using PM\#01, PM\#02, PM\#05, Aerosil200, and DQ25 at concentrations of $1 \mathrm{mg} / \mathrm{ml}$ in cell cul- ture medium and the more robust zeta dip cell with a platinum electrode were carried out. The latter comparison confirmed the values obtained with normal zeta cell measurements, thus suggesting no interference of the electrode types used with particle measurements. The particle size range for determinations of the zeta potential ranges between $5 \mathrm{~nm}$ and $10 \mu \mathrm{m}$. As $\mathrm{pH}$ is an important parameter influencing zeta potential, determination of the $\mathrm{pH}$ of selected samples (1 mg/ml Aerosil200, DQ25 and PM\#02) in cell culture medium with and without HEPES was performed with a $691 \mathrm{pH}$ Meter equipped with a Primatrode (Metrohm).

\section{Dynamic light scattering (DLS, Zetasizer)}

Measurement of hydrodynamic diameter was determined with a Zetasizer Nano ZS (Malvern Instruments $\mathrm{GmbH}$, Herrenberg, Germany) by dynamic light scattering (DLS) and values were reported as intensity, volume, and number weighted particle size distribution (PSD). The measureable size range for the Zetasizer using DLS ranges between $0.6 \mathrm{~nm}$ and $6 \mu \mathrm{m}$. Although the principle distribution value obtained from DLS measurement is an intensity weighted particle distribution, the volume and number weighted distributions are displayed as well. The scattering intensity of a particle is proportional to the sixth power of its diameter (from Rayleigh's approximation) (Chylek, 1986). As larger particles scatter much more light than smaller ones, the intensity of bigger particles may be overestimated. For example, the weighted intensity ratio of a $5 \mathrm{~nm}$ in comparison to a $50 \mathrm{~nm}$ particle is 1:1,000,000, whereas the weighted volume ratio of a $5 \mathrm{~nm}$ in comparison to a $50 \mathrm{~nm}$ particle is 1:1,000. In comparison, the weighted number size distribution is relatively independent of the particle size and thus, a priori, appears of greater value for particle size distributions in particle suspensions. However, the principle measurement is the intensity weighted particle distribution, which is then transformed to a volume or number weighted distribution. Both of the latter parameters contain a systematic transformation error. Measurement adjustments were set as follows: 2 measurements with 3 runs each $(10 \mathrm{sec}), 25^{\circ} \mathrm{C}$, measurement angle $173^{\circ}$ backscatter.

\section{Laser diffraction (Mastersizer)}

A continuous flow measurement of a particle suspension ranging between $20 \mathrm{~nm}$ and $2000 \mu \mathrm{m}$ diameter can be achieved with the Mastersizer (Mastersizer 2000, Malvern, Herrenberg) and provides particle volume size distributions. Similar to Zetasizer measurements, larger particles may provide an overestimation in the volume weighted size distribution. Employing similar transformation algorithms as for the Zetasizer (Mastersizer 2000 Malvern Application 5.60), the number weighted size distribution was determined. The refractive index of the material was set to 1.52 and 1.33 for the dispersant. Measurement adjustments were $15 \mathrm{sec}$ for measurement and background time and 15,000 measurement and background snaps. Sodium polyphosphate was used as diluent.

\footnotetext{
2 Zetasizer Nano User Manual, MAN0317 Issue 5.0 August 2009, ( ) Malvern Instruments Ltd. 2003 to 2008, 2009
} 


\section{Nanosight}

The Nanosight (LM20, NANOSIGHT, Wiltshire) visualizes and tracks particle movement over a certain time period based on Brownian movement of the particle. In conjunction with tracking analysis software and the individual particle tracks, a particle size distribution in a given suspension is obtained. The $\mathrm{Na}-$ nosight measurable particle diameters range between $10 \mathrm{~nm}$ and a maximum of 500 to $1000 \mathrm{~nm}$. Measurements were performed for polystyrene particles, DQ25, Aerosil200, PM\#01 as well as PM\#02 between 24 and $25^{\circ} \mathrm{C}$; because of limited sample material PM\#05 was not measured with Nanosight. Particles were tracked for $20 \mathrm{sec}$.

\section{PAH analysis in PM samples}

PM\#01, PM\#02, and PM\#05 were extracted according to DIN EN 15549 and resulted in Extract\#01, Extract\#02 and Extract\#05, respectively. Extraction was realized with Accelerated Solvent Extraction (ASE) with dichloromethane at $120^{\circ} \mathrm{C}$ and 100 bar (Dionex ASE 150 Extractor and Dionex SE 500 Concentrator). 16 US EPA PAHs (Mumtaz and George, 1995) were analyzed via adjacent GC-MS (Agilent gas chromatograph 6890 and Agilent mass selective detector 5975C).

\section{Salt, elementary (EC) and organic carbon (OC) analysis in PM samples}

Determination of EC and OC was realized by extraction and thermo-desorption on the basis of the German protocol VDI 2465-1, delivering the EC value, whereas a second subsample was analyzed for the total carbon (TC). The difference between TC and EC is defined as OC. Results are displayed as $\%$ of dry weight (dw). For analysis of anions the samples were extracted with water for 30 min under permanent shaking. The resulting water extracts were analyzed via isocratic ion chromatography (DIONEX ICS 90) and photometry (nitrite).

\section{Cell culture}

The human lung epithelial cell line A-549 was obtained from the German Collection of Microorganisms and Cell Cultures (Braunschweig, Germany) and cultured in DMEM/Ham's F12 supplemented with 10\% FBS, 25 mM HEPES and 1x P/S. Cells were passaged once to twice a week with a ratio of $1: 10$. PAH CALUX $^{\circledR}$ cells were obtained from BioDetection Systems and cultured according to the manufacturer's instructions using MEM Alpha modification, supplemented with 10\% FBS and $1 \mathrm{x} / \mathrm{S}$. All cells were maintained under standard conditions at $37^{\circ} \mathrm{C}, 100 \%$ humidity and $5 \% \mathrm{CO}_{2}$. Subculturing was performed twice a week with a ratio of 1:5. All cells were subcultured for a maximum of 20 passages.

\section{Cytotoxicity assay}

Cytotoxicity was assessed using the MTT assay. MTT (3-(4,5-dimethylthiazol-2-yl)-2,5-diphenyltetrazolium bromide) is transformed by enzymes of the mitochondria and the endoplasmic reticulum to formazan, which is detected spectrophotometrically. In short, 7,000 cells/well were seeded in 96 well plates. After $48 \mathrm{~h}$ cells were treated with different concentrations of PM $\left(0.01,0.04,0.1,0.5,2,8\right.$, and $\left.25 \mu \mathrm{g} / \mathrm{cm}^{2}\right)$. After
$48 \mathrm{~h}$ of exposure, $10 \mu 1 \mathrm{MTT}(5 \mathrm{mg} / \mathrm{ml})$ per $100 \mu 1$ medium was added to the wells and incubated for another $1.5 \mathrm{~h}$ in the incubator. Supernatant was discarded and $100 \mu \mathrm{l} /$ per well solubilization solution (95\% isopropanol, $5 \%$ formic acid) was added and the plates were shaken on a plate shaker for 5-10 min. Absorbance was measured at $550 \mathrm{~nm}$. Because of artifacts in measurement due to the intense absorbance characteristics of the black particles, a reference plate for each PM was exposed with the same concentrations but without cells. The resulting absorbance values were subtracted from the absorbance values obtained for the exposed cells.

\section{PAH CALUX ${ }^{\circledR}$ assay}

The PAH CALUX ${ }^{\circledR}$ (chemically activated luciferase expression) cell line is a genetically engineered rat hepatoma cell line from BioDetection Systems. Upon exposure to Ah receptor agonists, e.g., PAHs, dioxins and dioxin-like PCBs a luciferase signal is produced which is proportional to the quantity of receptor binding. The assay was performed according to the manufacturer's protocol. Briefly, 40,000 cells/well were seeded on 96-well plates. After $24 \mathrm{~h}$, the cells were treated with conditioned medium containing either the benzo[a]pyrene $(\mathrm{B}[\mathrm{a}] \mathrm{P})$ reference in different concentrations (0 M, 1.25E-09 M, 1.25E-08 M, 3.75E$08 \mathrm{M}, 1.25 \mathrm{E}-07 \mathrm{M}, 3.75 \mathrm{E}-07 \mathrm{M}, 1.25 \mathrm{E}-06 \mathrm{M}, 1.25 \mathrm{E}-05 \mathrm{M}, 1.25 \mathrm{E}-$ $04 \mathrm{M}, 1.25 \mathrm{E}-03 \mathrm{M}$ ) or the sample in three technical replicates. Plates were incubated for $4 \mathrm{~h}$ at $37^{\circ} \mathrm{C}, 5 \% \mathrm{CO}_{2}$. After exposure the cells were lysed by addition of $80 \mu \mathrm{l}$ Steady Glo ${ }^{\circledR}$ per well. After 5-10 min $70 \mu \mathrm{l}$ of the lysate was transferred into a white 96-well flat bottom plate and luminescence was measured. For analysis, the value for the vehicle control (0.8\% DMSO) was subtracted from all other values measured, and a standard doseresponse curve was calculated using the values measured for the $\mathrm{B}[\mathrm{a}] \mathrm{P}$ treatment in the different concentrations. Luminescence values measured with the cells treated with the PM samples or extracts were normalized by dividing them by the maximum value obtained from the $\mathrm{B}[\mathrm{a}] \mathrm{P}$ treatment on the same plate and given as a percentage of this maximum value.

\section{Statistical analysis}

All results are expressed as the mean \pm standard error of means for $\geq$ three replicates unless otherwise noted. All pair-wise multiple comparisons were analyzed with the unpaired t-test while multiple comparisons were carried out via a one-way ANOVA followed by a Bonferroni post-test as indicated (Graph Pad Prism ${ }^{\circledR}$ version 5.04.)

\section{Results}

\section{Scanning electron microscopy (SEM)}

Particulate matter samples as well as the reference (polystyrene and quartz) particles were first analyzed via SEM in a dry state. SEM images revealed a spherical shape for all three polystyrene particles (non-fluorescent), and confirmed their round shape and size (Fig. 1A-C). Small deviations in diameter were observed, albeit a coefficient of variance of $15 \%, 8 \%$ and $3 \%$ for the $50 \mathrm{~nm}, 200 \mathrm{~nm}$, and $1,000 \mathrm{~nm}$ particles, respectively, is possible 


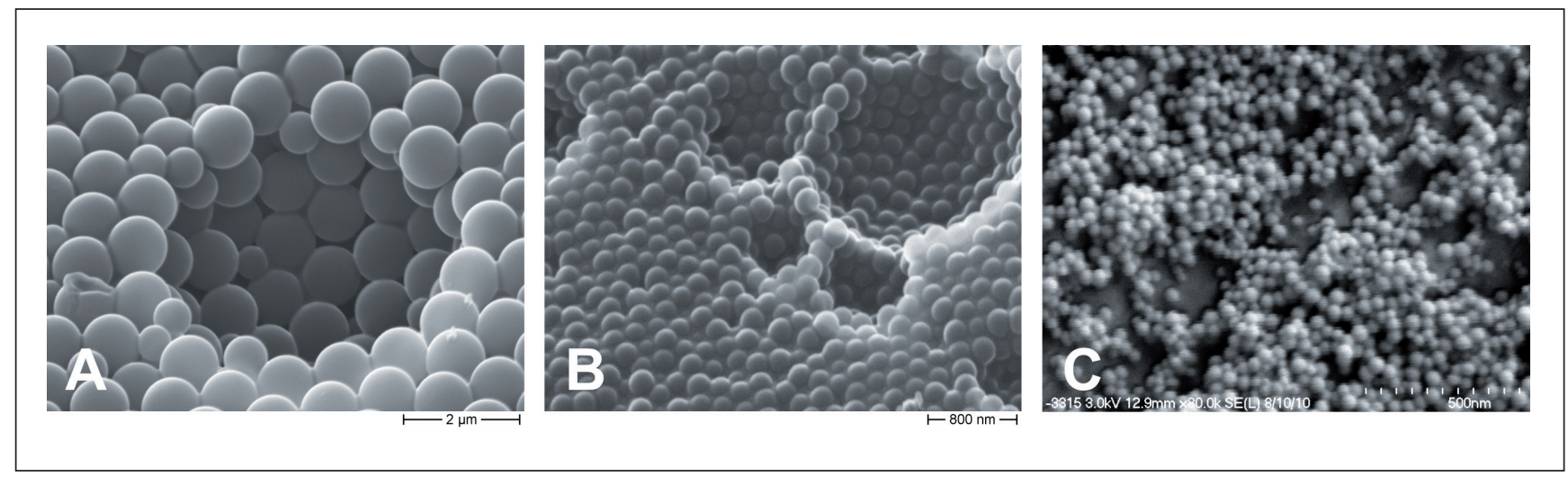

Fig. 1: Representative SEM images of non-fluorescent polystyrene particles with a diameter of 1,000 nm (A), $200 \mathrm{~nm}(B)$, and $50 \mathrm{~nm}(\mathrm{C})$

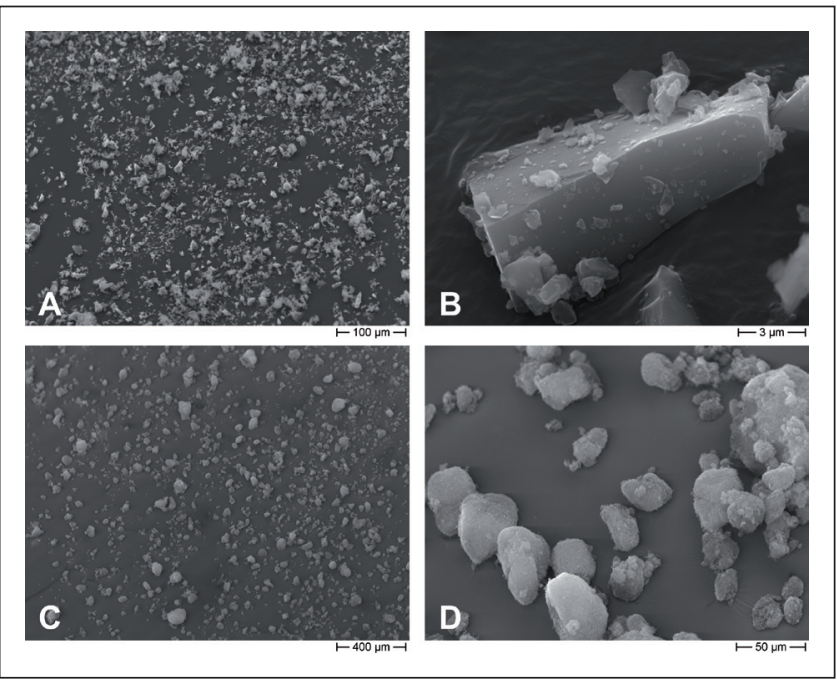

Fig. 2: Representative SEM images of DQ25 (A, B) and Aerosil200 (C, D)

according to the manufacturer. The comparison of the different polystyrene particles demonstrated that diameters $\leq 50 \mathrm{~nm}$ are difficult to characterize via the SEM types used for this study because of limited size resolution. In contrast, the two quartz samples showed a less homogeneous particle size distribution (Fig. 2). The particle size of DQ25 largely corresponded to the diameter/size defined by the manufacturers, whereas Aerosil200 particles exceeded the manufacturer's guaranteed diameter of $12 \mathrm{~nm}$, i.e., some particles with diameters ranging between 10 $\mu \mathrm{m}$ and $70 \mu \mathrm{m}$. As expected, PM\#01, \#02, and \#05 (Fig. 3) presented an inhomogeneous particle size distribution, whereas PM\#02 generally contained larger particle aggregates than PM\#01 and PM\#05.

\section{Light microscopy}

No aggregation was observed for fluorescent and non-fluorescent polystyrene particles, irrespective of the medium (water or cell culture media) and particle diameters. Similarly, no visible differences were observed for DQ25 or the PM samples suspended in media and water (data not shown). In contrast,

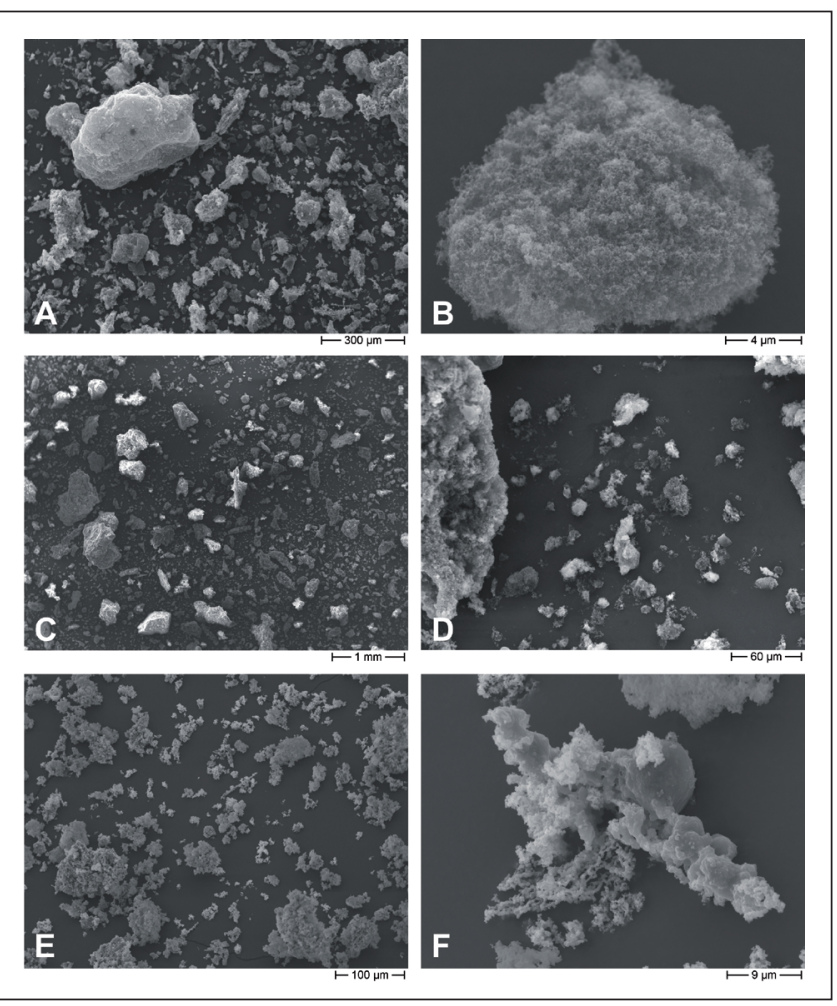

Fig. 3: Representative SEM images of PM\#01 (A, B), PM\#02 (C, D), and PM\#05 (E, F)

Aerosil200 suspensions in media resulted in more and larger aggregates than water suspensions (Fig. 4).

\section{Zeta potential and $\mathrm{pH}$}

In contrast to the light microscopic observations, determinations of the zeta potential suggested an increased trend for polystyrene particles to aggregate in cell culture media, whereas homogeneous suspensions were found in water suspensions (Fig. 5A, S1). Indeed, a significant difference in zeta potential between cell culture media and water was observed for all particle suspensions, irrespective of the particle type and thus sample homogeneity, suggesting a generally higher suspen- 

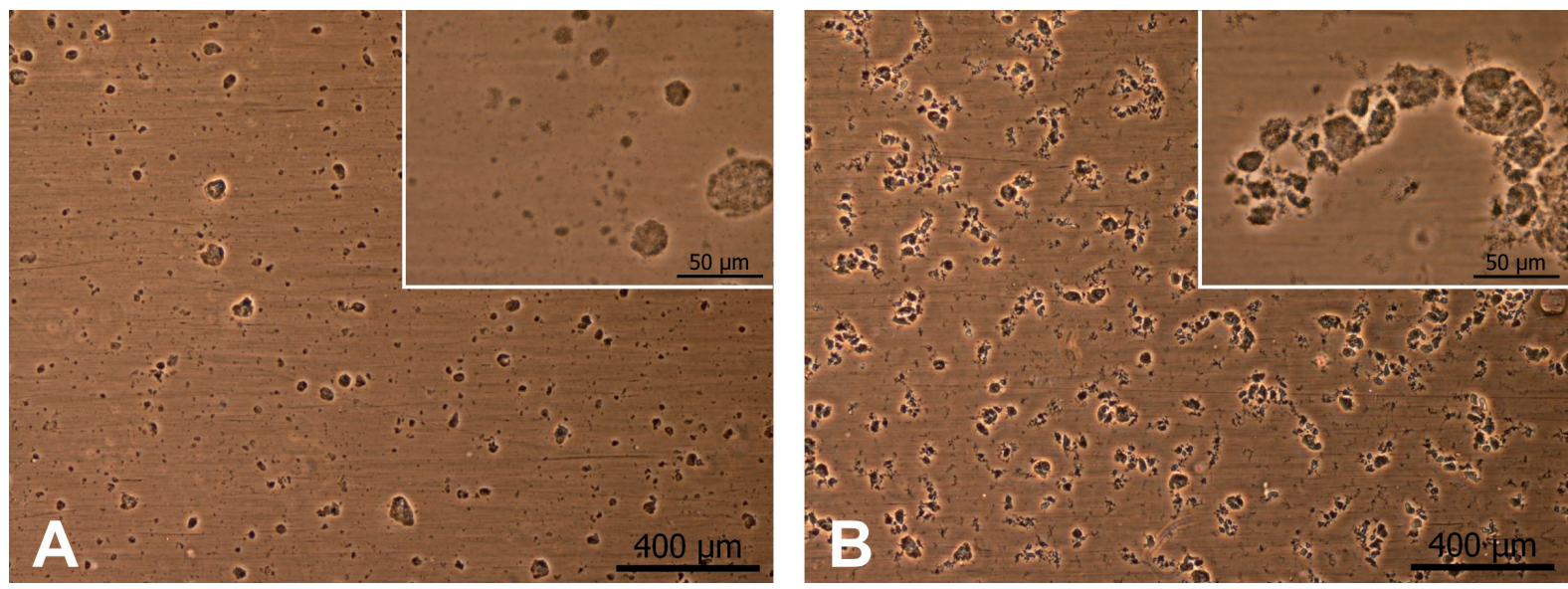

Fig. 4: Representative images of Aerosil200 suspensions in ultrapure water (A) and cell culture medium (B)

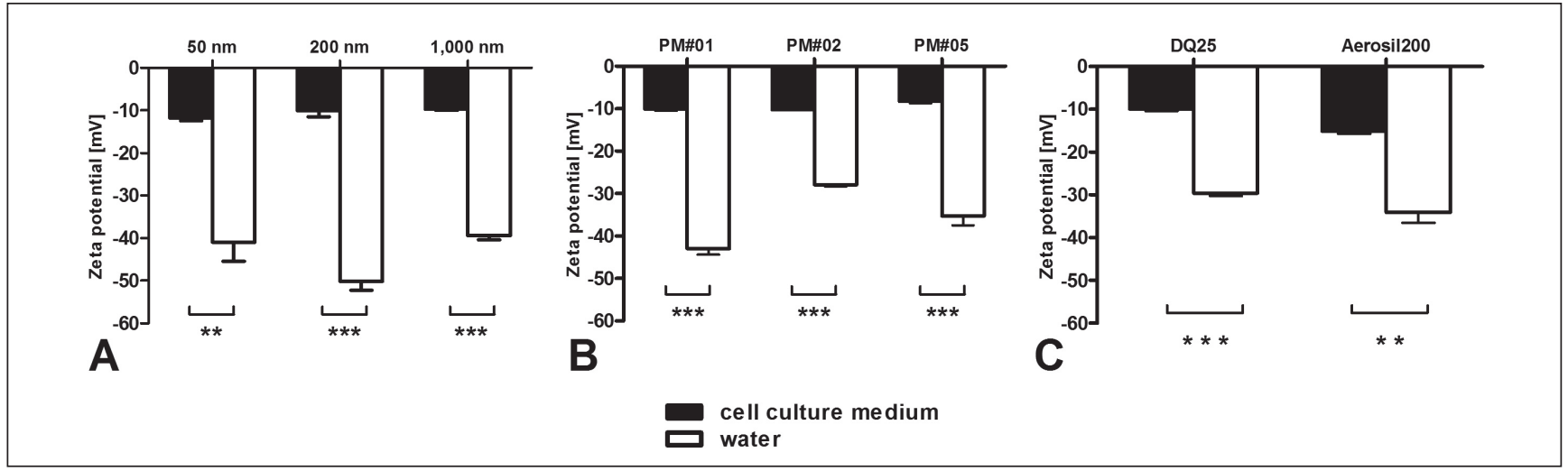

Fig. 5: Zeta potential measurements of 50, 200, and 1,000 nm non-fluorescent polystyrene particles (A), $1 \mathrm{mg} / \mathrm{mI} \mathrm{PM \# 01,} \mathrm{PM \# 02,}$ and PM\#05 (B), DQ25, and Aerosil200 (C)

Mean \pm SEM is shown $(n=3)$. Unpaired t-test, ${ }^{*} p<0.05,{ }^{* *} p<0.01,{ }^{* * *} p<0.001$

sion stability and lower tendency to form particle aggregates in water (zeta potential around $<-30 \mathrm{mV}$ ) than in cell culture media (Fig. 5A, Fig. S1). Control measurements of water and RPMI culture medium (Fig. 6) without particles also revealed a significantly higher zeta potential for medium $(-4.41 \mathrm{mV})$ than for water $(-9.53 \mathrm{mV})$. Zeta potential measurements of PM\#02, DQ25 and Aerosil200 in cell culture medium (-HEPES) resulted in $-11.3 \mathrm{mV} \pm 1.1(\mathrm{n}=3),-12.4 \mathrm{mV} \pm 0.1(\mathrm{n}=3)$, and $-17 \mathrm{mV} \pm 3$ $(\mathrm{n}=3)$, respectively. The comparison values obtained for PM\#02, DQ25 and Aerosil200 in cell culture medium (+HEPES) normally used for particle characterization, i.e., $-10.2 \mathrm{mV} \pm 0.1$ $(\mathrm{n}=3),-9.9 \mathrm{mV} \pm 0.8(\mathrm{n}=3)$, and $-15.1 \mathrm{mV} \pm 1.1 \quad(\mathrm{n}=3)$, respectively, demonstrated a negligible influence of HEPES on the zeta potential determinations.

Measurement of RPMI (-HEPES) resulted in a $\mathrm{pH}$ value of $7.56 \pm 0.03(n=3)$. The addition of HEPES led to a slight decrease in $\mathrm{pH} 7.27 \pm 0.03(\mathrm{n}=3)$, also visible by slight color change of phenol red. Upon addition of PM\#02, DQ25 and Aerosil200 to RPMI (-HEPES) a slight, but not significant increase in $\mathrm{pH}$ was observed $7.75 \pm 0.11(n=3), 7.85 \pm 0.28(n=3)$, and 8.23 $\pm 0.28(\mathrm{n}=3)$. The $\mathrm{pH}$ of the RPMI (+HEPES) did not change significantly upon addition of PM\#02 (7.24 $\pm 0.07(n=3))$, Aerosil200 $(7.45 \pm 0.05(n=3))$, or DQ25 $(7.38 \pm 0.06(n=3))$. The $\mathrm{pH}$ measurement of ultrapure water without any particles was not possible, because of quasi-absence of ions, however the theoretical $\mathrm{pH}$ value is about 6.998 (Nora et al., 2002). The observation that comparable zeta potentials were registered for all particle types in RPMI (Fig. S1, see supplementary data at www.altex-edition.org) is presumably due to the $\mathrm{pH}$ $(7.27 \pm 0.11(\mathrm{n}=3))$ of the RPMI. As $\mathrm{pH}$ is known to influence zeta potential values (Berg et al., 2009), a stable $\mathrm{pH}$ will most likely result in similar zeta potential readings, whereas zeta potentials obtained in water are more strongly influenced by the respective particle composition. Cell culture media are buffered to prevent $\mathrm{pH}$ changes. The RPMI medium used in this study, additionally contained HEPES - a buffer substance - thus possi- 


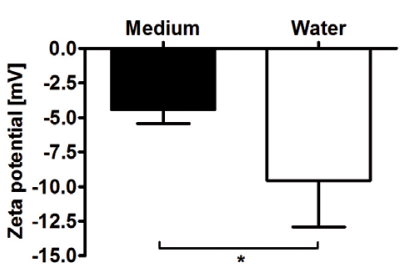

A

Size Distribution by Number

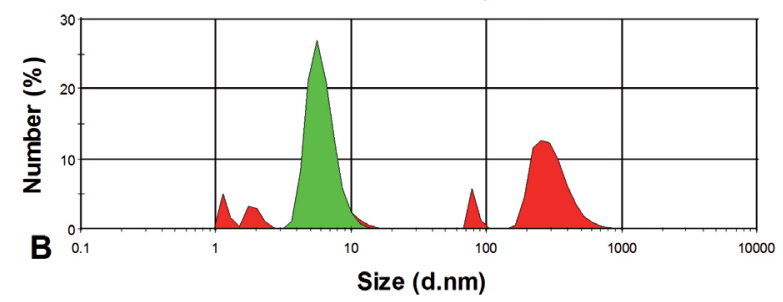

Fig. 6: Zeta potential (A) and Zetasizer number weighted particle size distribution measurements $(B)$ of ultrapure water (red area) and medium (green area) without any particles The frequency curves are shown. Mean \pm SEM is shown $(n \geq 5)$. Unpaired t-test, ${ }^{*} p<0.05$.

bly stabilizing the $\mathrm{pH}$ independent of the particle type suspended in the media. Comparative measurements with RPMI supplemented with FBS and P/S (-HEPES) resulted in a slightly, but insignificantly, lower zeta potential and $\mathrm{pH}(7.56 \pm 0.03(\mathrm{n}=3))$, compared to RPMI (+HEPES) $(7.27 \pm 0.11(n=3))$.

\section{Particle Size Distribution measured with Zetasizer}

Zetasizer measurements of the suspension media showed that medium components (with/without HEPES) resulted in a peak at around $6 \mathrm{~nm}$, whereas the ultrapure water without any particles had several small peaks between 1 and $250 \mathrm{~nm}$, potentially resulting from impurities (Fig. 6B). A trend for particle suspension aggregation in cell culture media, although not as pronounced, was observed when polystyrene particles were analyzed via the Zetasizer particle size distribution (Fig. 7, 8), generally displaying larger particle diameters than expected from the particle diameters defined via SEM. The fluorophore in the polystyrene particles had no significant influence on measured particle size distribution (Fig. 8). All polystyrene particle measurements resulted in one single peak, except for the measurement of the $50 \mathrm{~nm}$ non-fluorescent beads in medium. The second peak at $\sim 13 \mathrm{~nm}$ might result from media components (Fig. 7B). The trend to larger measured particle diameters, i.e., to form aggregates, was more pronounced for Aerosil200 compared to crystalline quartz. Specifically, two peaks at 1,484 and $1,106 \mathrm{~nm}$ were visible for Aerosil200 in cell culture medium, whereas the frequency curve for the water suspensions had two peaks at 712 and $105 \mathrm{~nm}$ (Fig. 9). Statistical analysis of mean particle sizes revealed significant differences between water and cell culture medium (Fig. 10B, S2D-F). Similarly, larger mean particle diameters were recorded in cell culture media than in

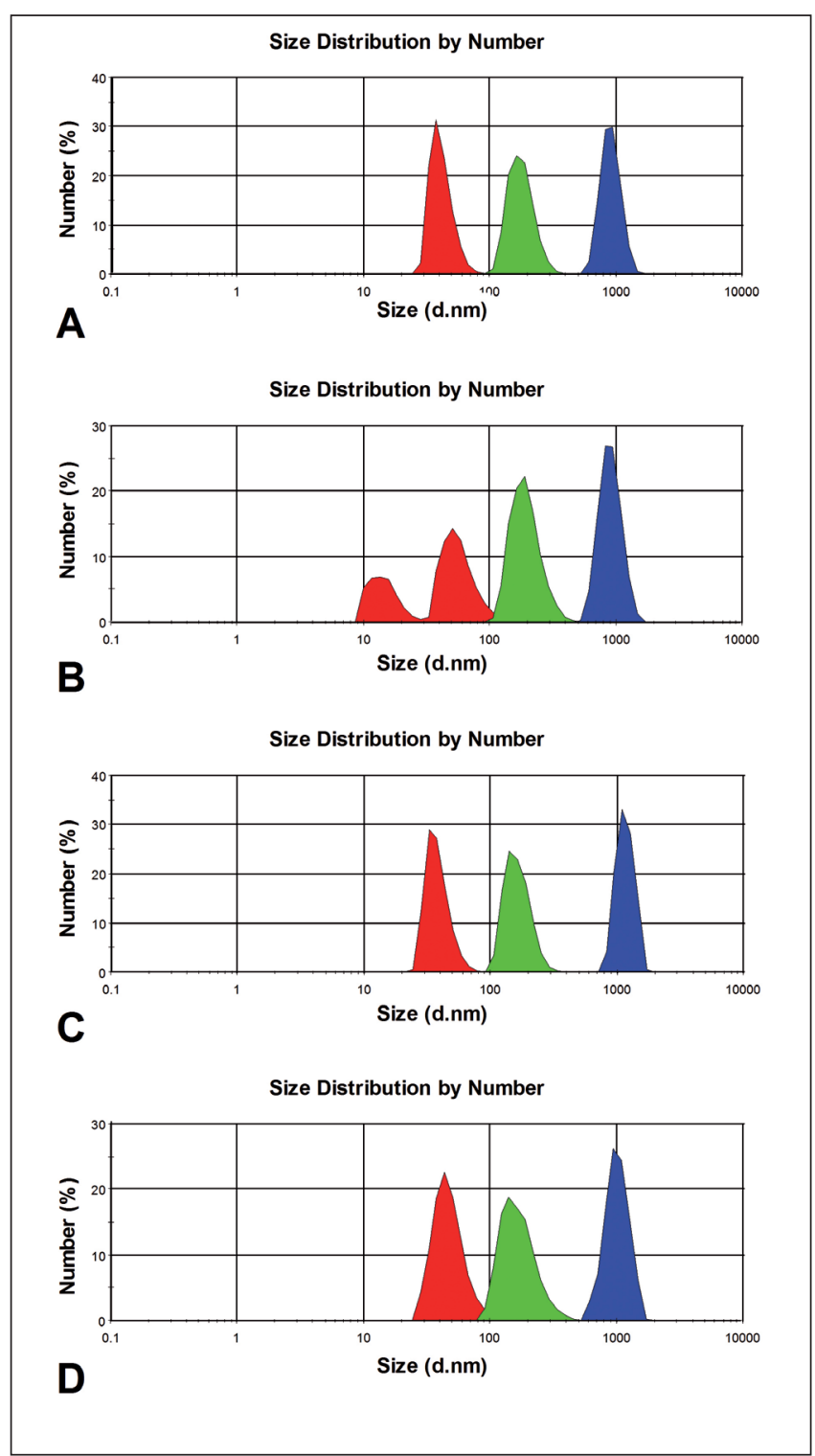

Fig. 7: Zetasizer measurements of number weighted particle size distribution for non-fluorescent $(A, B)$ and fluorescent particles (C, D) in water $(A, C)$ and cell culture medium $(B, D)$ for $50 \mathrm{~nm}$ (red area), $200 \mathrm{~nm}$ (green area), and 1,000 nm (blue area) particles

Mean of three independent measurements

water for PM\#01 but not for PM\#02 and PM\#05 (Fig. 11, 12, S3), suggesting a slightly higher tendency for PM\#01 to form aggregates in comparison to PM\#02 and PM\#05.

Particle size distribution measured with Mastersizer

Based on the detection capabilities of the Mastersizer (Tab. 4), measurement of the $200 \mathrm{~nm}$ and $1,000 \mathrm{~nm}$ polystyrene particle suspensions reflected their expected diameters. Considering the mean particle size, the diameters of the $50 \mathrm{~nm}$ particles were partially overestimated (Tab. 5, Fig. 13A), irrespective of whether a volume or number weighted mean was calculated. This is con- 

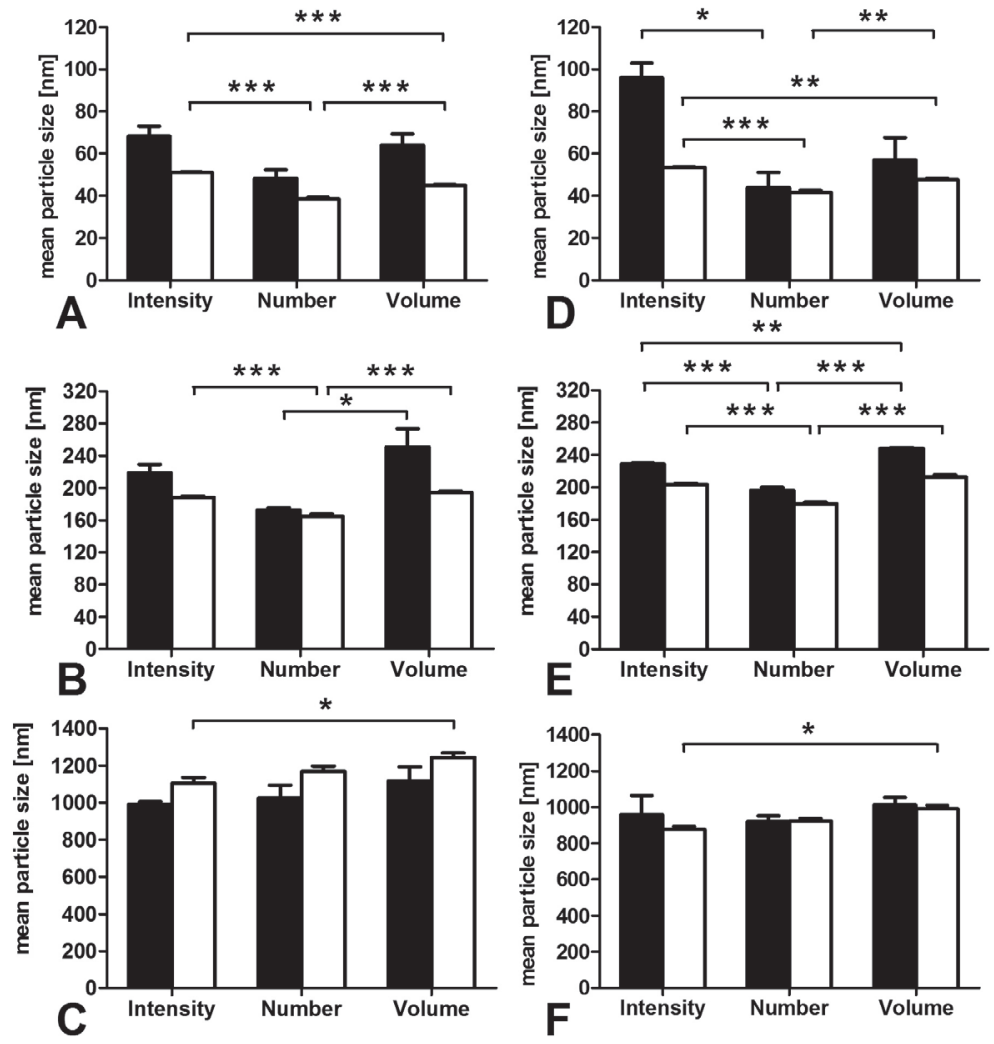

cell culture medium water
Fig. 8: Zetasizer measurements of particle size distribution for fluorescent $(A-C)$ and nonfluorescent particles (D-F) in water and cell culture medium for $50 \mathrm{~nm}(\mathrm{~A}, \mathrm{D}), 200 \mathrm{~nm}$ (B, E), and $1,000 \mathrm{~nm}(C, F)$ particles The intensity weighted, number weighted, and volume weighted means are shown. Mean \pm SEM is shown $(n=3)$. One-way ANOVA with Bonferroni post-test was used to compare intensity, number, and volume weighted means in cell culture medium and water. ${ }^{*} \mathrm{p}<0.05$, ${ }^{* *}$ $p<0.01,{ }^{* \star *} p<0.001$

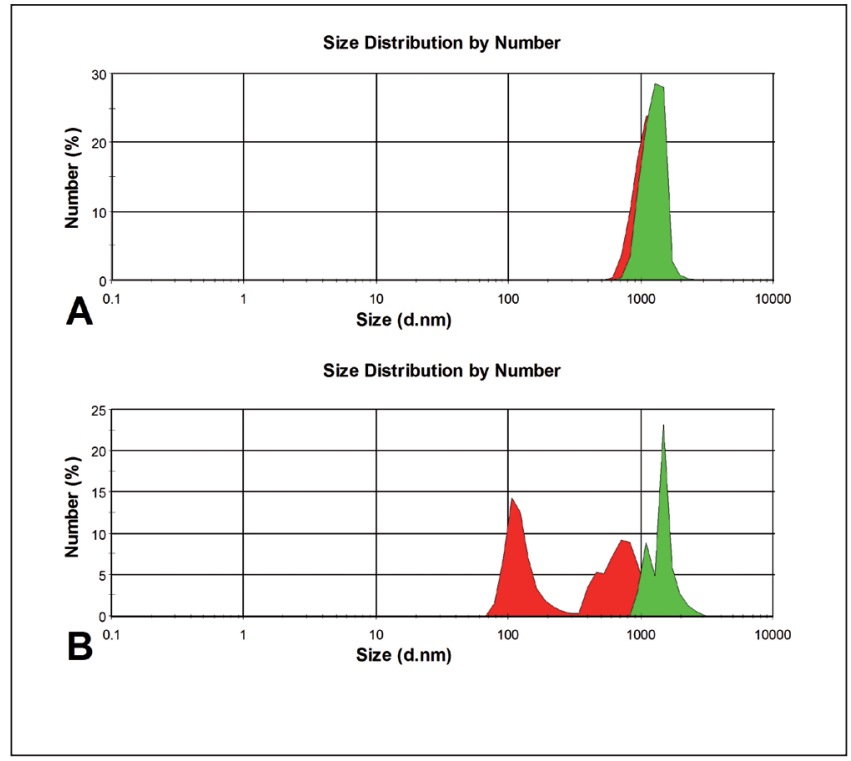

Fig. 9: Zetasizer measurements of particle size distribution of $1 \mathrm{mg} / \mathrm{ml} \mathrm{DQ25} \mathrm{(A)} \mathrm{and} \mathrm{Aerosil200} \mathrm{(B)} \mathrm{in} \mathrm{water} \mathrm{(red} \mathrm{area)} \mathrm{and}$ cell culture medium (green area)

The number weighted particle size distribution is shown. Mean of three independent measurements.

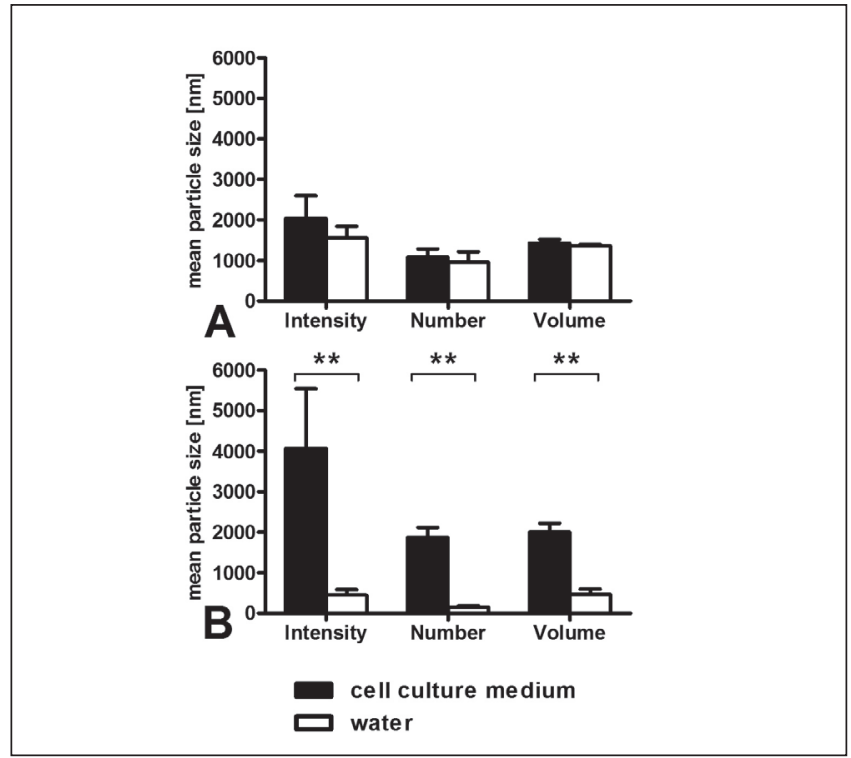

Fig. 10: Zetasizer measurements of particle size distribution of $1 \mathrm{mg} / \mathrm{ml} \mathrm{DQ25} \mathrm{(A)} \mathrm{and} \mathrm{Aerosil200} \mathrm{(B)} \mathrm{in} \mathrm{water} \mathrm{and} \mathrm{cell}$ culture medium

The intensity weighted, number weighted, and volume weighted means for Aerosil200 and DQ25 are shown, respectively. Mean ${ }_{ \pm} \mathrm{SEM}$ is shown $(n=3)$. Unpaired t-test, ${ }^{*} \mathrm{p}<0.05,{ }^{* *} p<0.01$. 


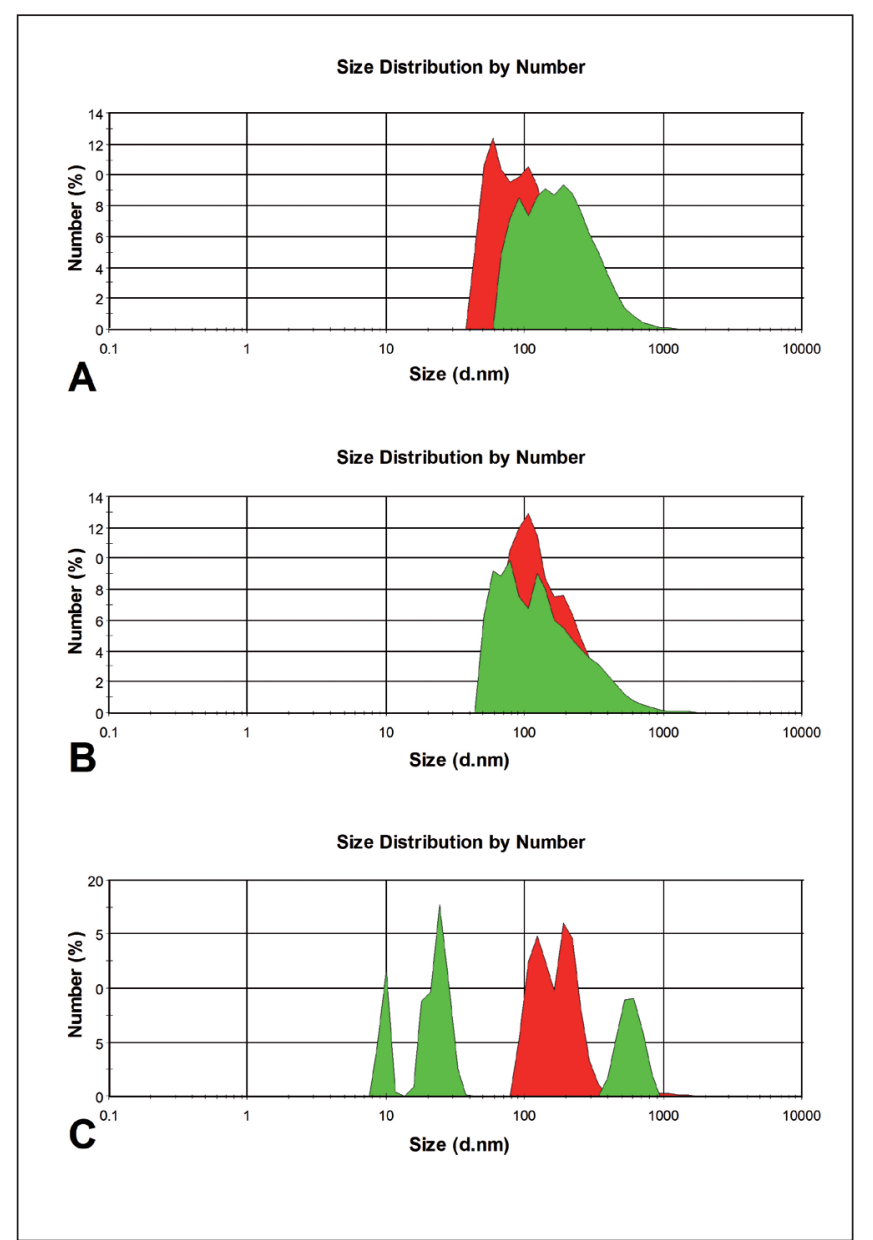

Fig. 11: Zetasizer measurements of particle size distribution of $1 \mathrm{mg} / \mathrm{ml} \mathrm{PM \# 01(A),} \mathrm{PM \# 02} \mathrm{(B),} \mathrm{and} \mathrm{PM \# 05} \mathrm{(C)} \mathrm{in} \mathrm{water} \mathrm{(red}$ area) and cell culture medium (green area).

The number weighted particle size distribution is shown. Mean of three independent measurements.

sistent with the frequency curves obtained from the Mastersizer, where the $50 \mathrm{~nm}$ particles in general had a broader size distribution than the 200 and 1,000 nm particles (Fig. 14). The calculated undersize values revealed that $90 \%$ of the non-fluorescent $50 \mathrm{~nm}, 200 \mathrm{~nm}$, and 1,000 nm polystyrene particles in medium are below $86.66 \mathrm{~nm}, 236.0 \mathrm{~nm}$ and $947.0 \mathrm{~nm}$, respectively (Tab. 5). Similarly, the mean diameter of Aerosil200 was severely overestimated (Fig. 15A-B, S4A-B), while the diameter of DQ25 was correctly determined (Fig. 15A-B, S4C-D). The latter could suggest aggregation of Aerosil200 in cell culture media as well as in water, as well as inhomogeneity of the diameters of the suspended material (Fig. 16B, Tab. 6). Notably, smaller differences with regard to aggregation between suspensions in cell culture media and water were observed for DQ25, where the peaks for the medium and the water suspensions were between 405-550 $\mathrm{nm}$ and 348-473 nm, respectively (Fig. 16A, Tab. 6). The number weighted Mastersizer measurements for the PM samples suggested small particle sizes for PM\#01, having a peak between 57-77

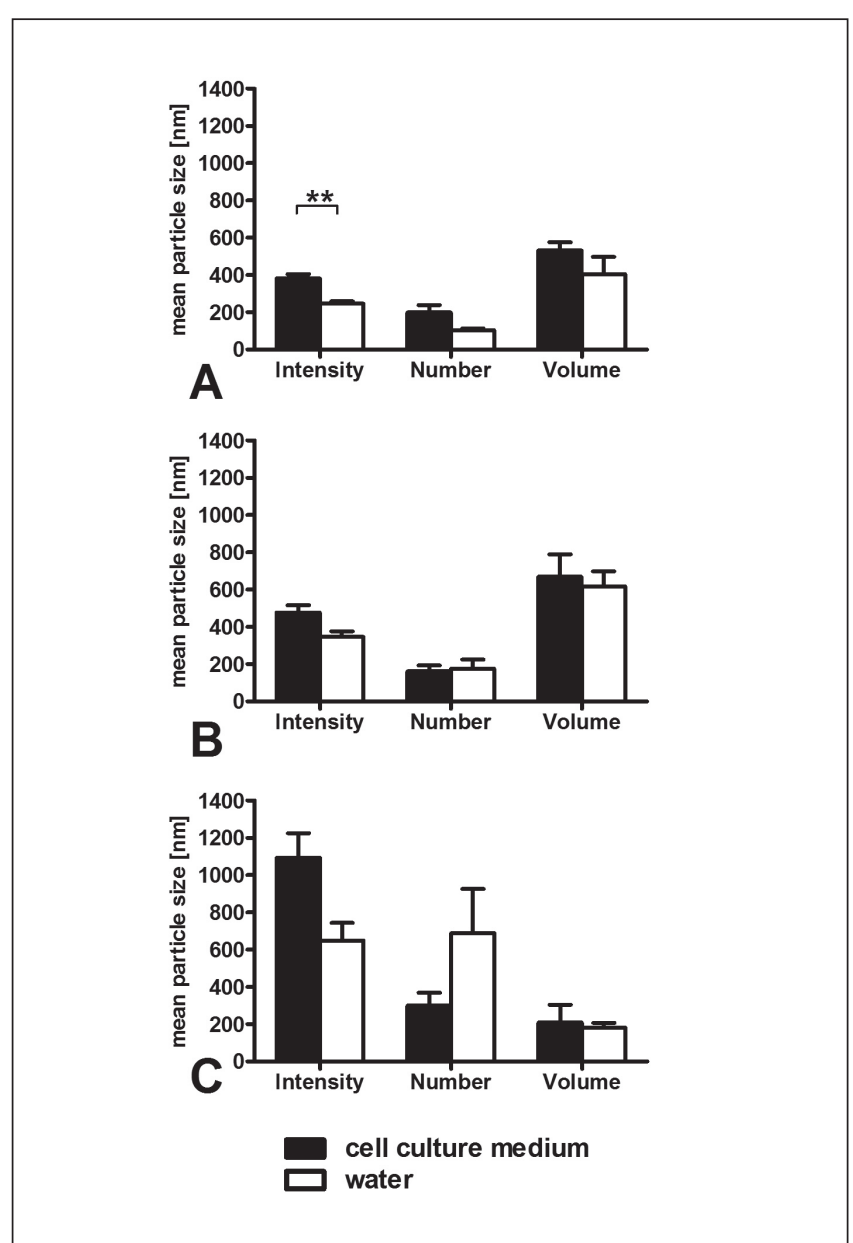

Fig. 12: Zetasizer measurements of particle size distribution of $1 \mathrm{mg} / \mathrm{ml} \mathrm{PM \# 01} \mathrm{(A),} \mathrm{PM \# 02} \mathrm{(B),} \mathrm{and} \mathrm{PM \# 05} \mathrm{(C)} \mathrm{in} \mathrm{water} \mathrm{and}$ cell culture medium

The intensity weighted, number weighted and volume weighted means for $\mathrm{PM \# 01}$ and $\mathrm{PM \# 02}$ are shown, respectively. Mean ${ }_{ \pm}$SEM is shown $(n=3)$. Unpaired t-test, ${ }^{* *} p<0.01$.

nm, whereas PM\#02 peaked between 190-308 nm and PM\#05 between 278-428 nm (Fig. 17). Similar to Zetasizer, the Mastersizer measurements could not definitively confirm a higher trend to aggregation of PM\#01, PM\#02, and PM\#05 in cell culture media than in water (Tab. 6, Fig. 17, 18C-D, S5B).

\section{Particle size distribution measured with Nanosight}

Measurement of the 1,000 nm polystyrene particles with $\mathrm{Na}$ nosight appeared critical, lead to conflicting results and thus particle sizes were effectively undeterminable as this size was at the resolution limit of the Nanosight. In contrast, measurements obtained for the $200 \mathrm{~nm}$ polystyrene particles correctly reflected the expected diameters. The $50 \mathrm{~nm}$ beads appear to form aggregates of at least two particles, because diameters up to $100 \mathrm{~nm}$ were measured, as already reported for colloidal gold nanoparticles in human blood (Dobrovolskaia et al., 2009). Aerosil200 suspension revealed particle sizes in water of approx. $25 \mathrm{~nm}$ while double this diameter was determined for suspen- 


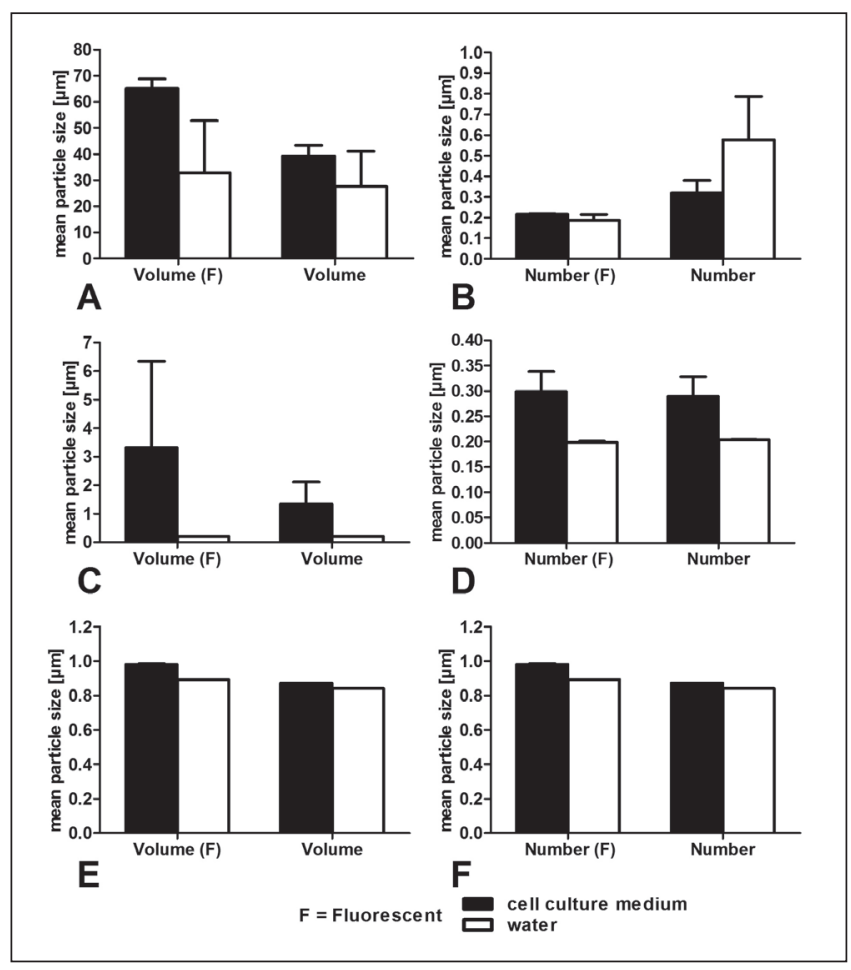

Fig. 13: Mastersizer measurements of mean particle size for fluorescent (indicated with $F$ ) and non-fluorescent polystyrene particles with $50 \mathrm{~nm}$ (A, B), $200 \mathrm{~nm}$ (C, D), and $1,000 \mathrm{~nm}(E, F)$ in water and cell culture medium Volume weighted and number weighted means are shown as indicated. Mean \pm SEM is shown $(n=3)$.

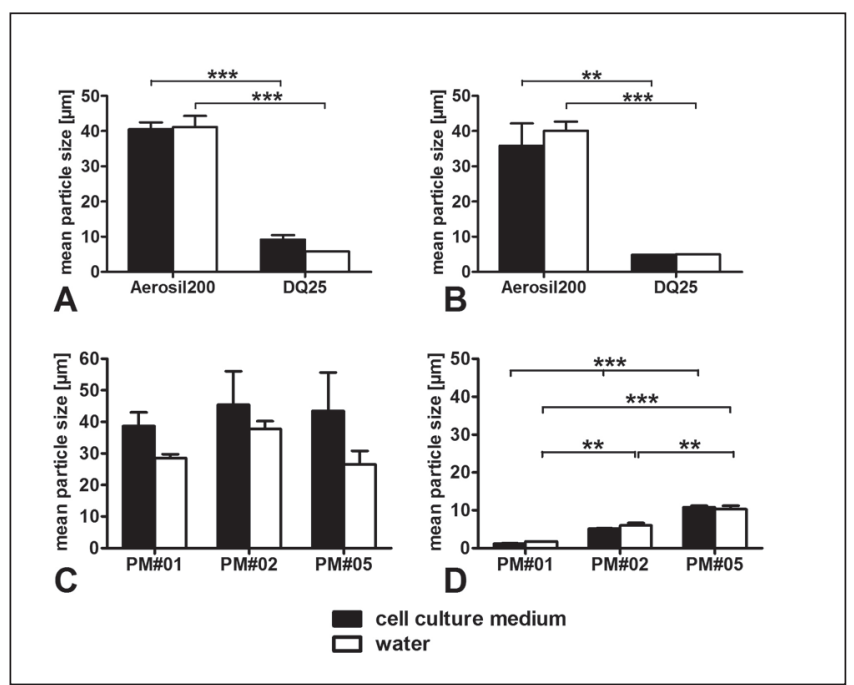

Fig. 15: Mastersizer measurements of Aerosil200 and DQ25 of volume (A) and number (B) weighted mean particle size in water and cell culture medium and volume (C) and number (D) weighted mean particle size of PM\#01, PM\#02 and PM\#05 in water and cell culture medium

Volume weighted and number weighted means are shown as indicated. Mean $\pm S E M$ is shown $(n \geq 3)$. Unpaired t-test used for $A$ and $B$, One-way ANOVA with Bonferroni post-test for $C$ and $D$; *** $\mathrm{p}<0.001,{ }^{* *} \mathrm{p}<0.01$.

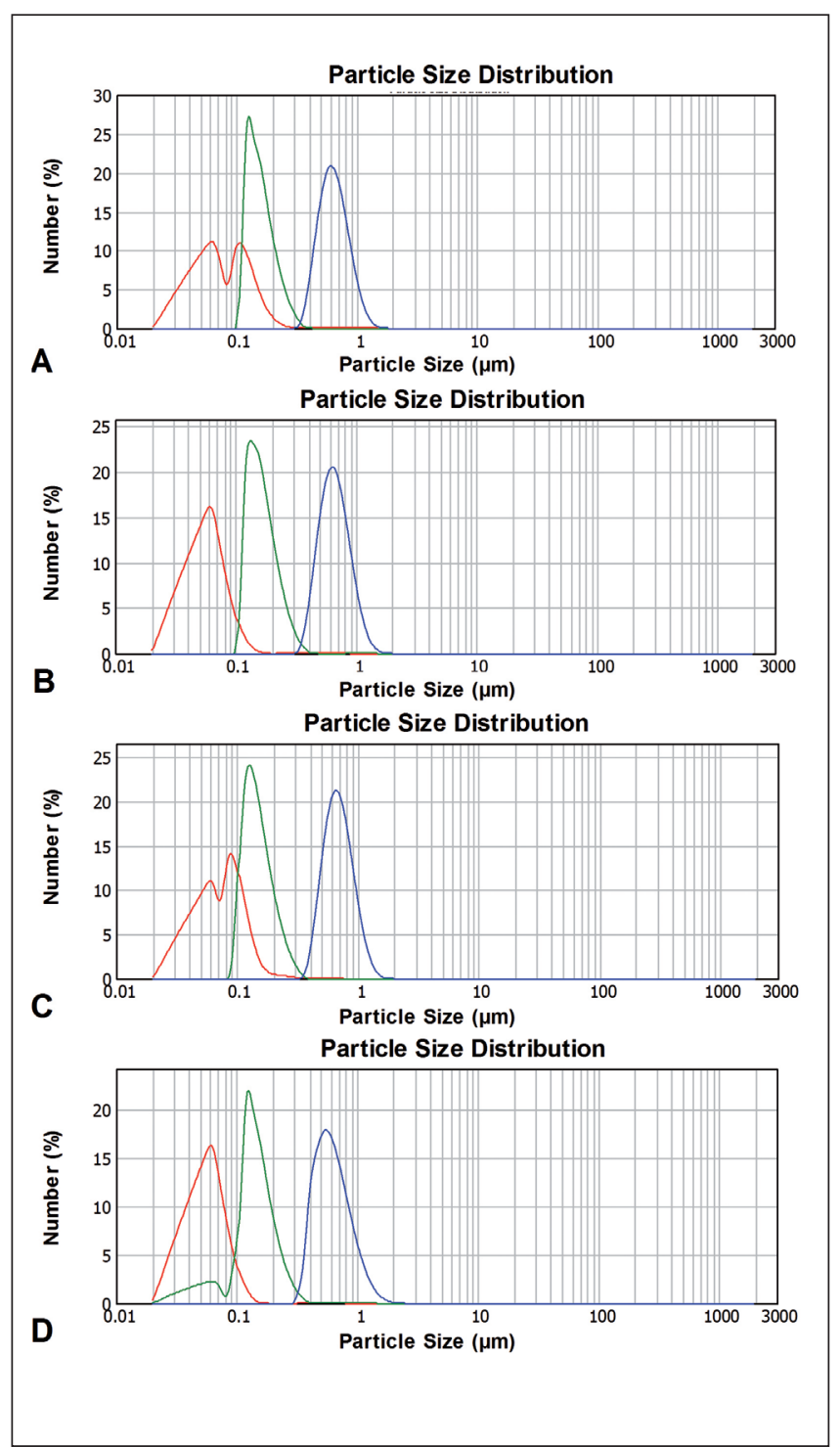

Fig. 14: Mastersizer measurements of number weighted particle size distribution for non-fluorescent $(A, B)$ and fluorescent particles $(C, D)$ in water $(A, C)$ and cell culture medium (B, D) for $50 \mathrm{~nm}$ (red line), $200 \mathrm{~nm}$ (green line), and $1,000 \mathrm{~nm}$ (blue line) particles

Mean of three independent measurements. 


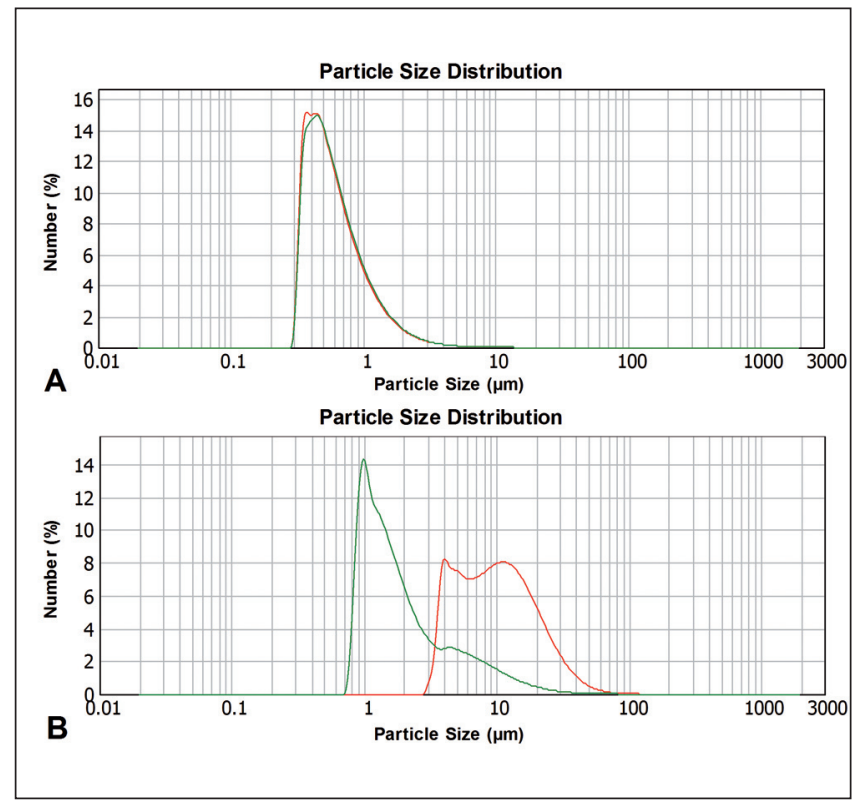

Fig. 16: Mastersizer measurements of number weighted particle size distribution for DQ25 (A) and Aerosil200 (B) in water (red line) and cell culture medium (green line) Mean of three independent measurements.

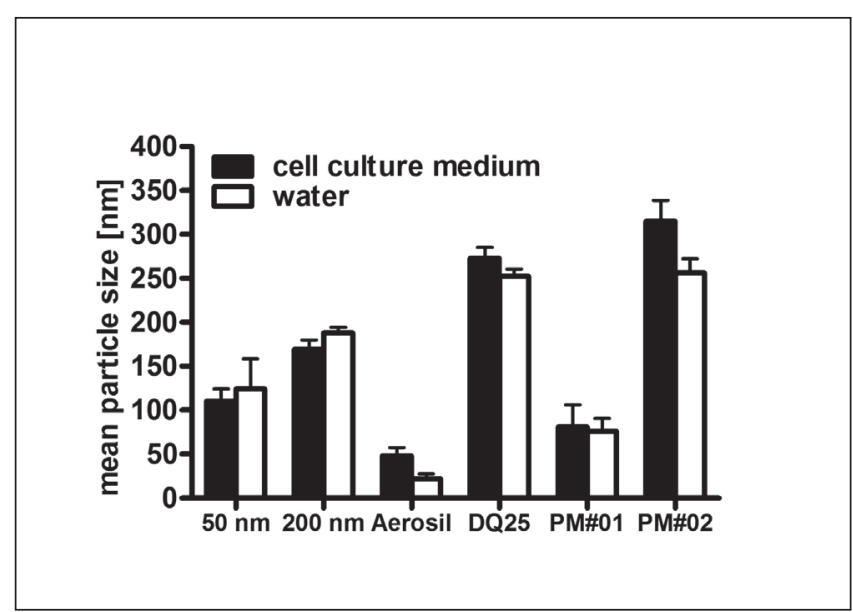

Fig. 18: Summary of Nanosight measurements for the different particle types in water and cell culture medium Mean \pm SEM is shown $(n \geq 3)$.
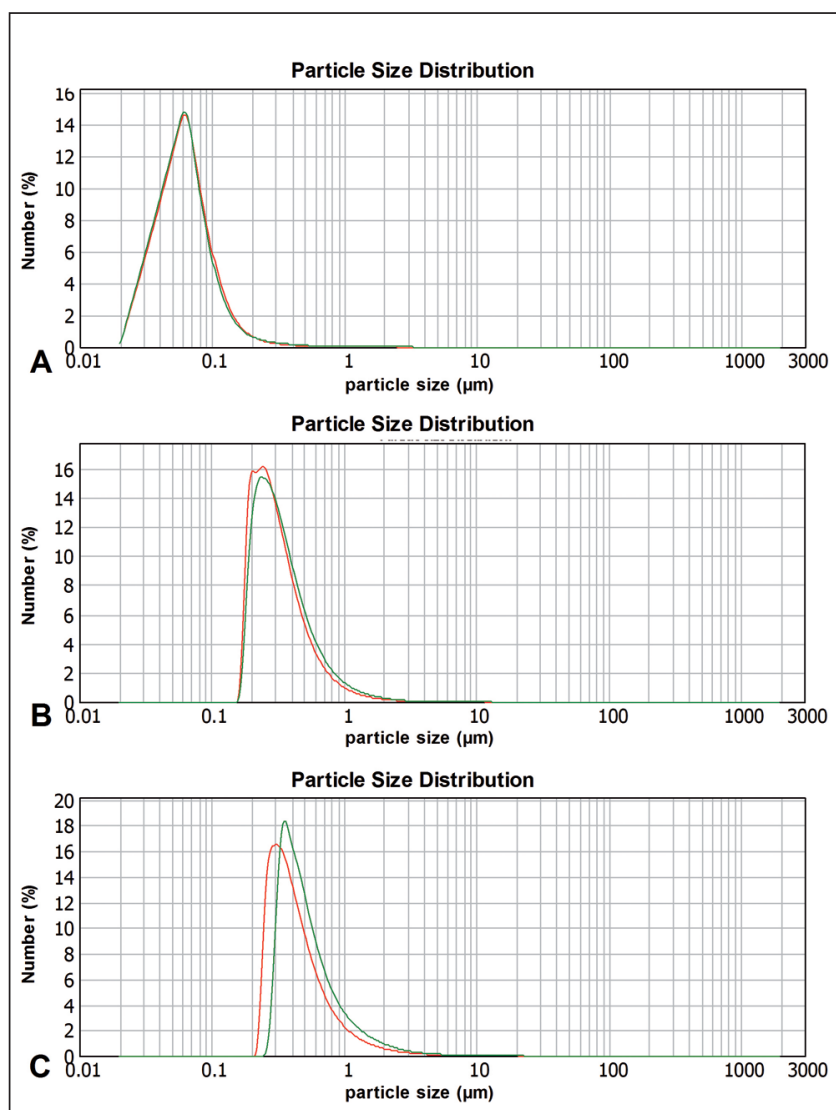

Fig. 17: Mastersizer measurements of number weighted particle size distribution for PM\#01 (A), PM\#02 (B), and PM\#05 in water (red line) and cell culture medium (green line) Mean of three independent measurements.

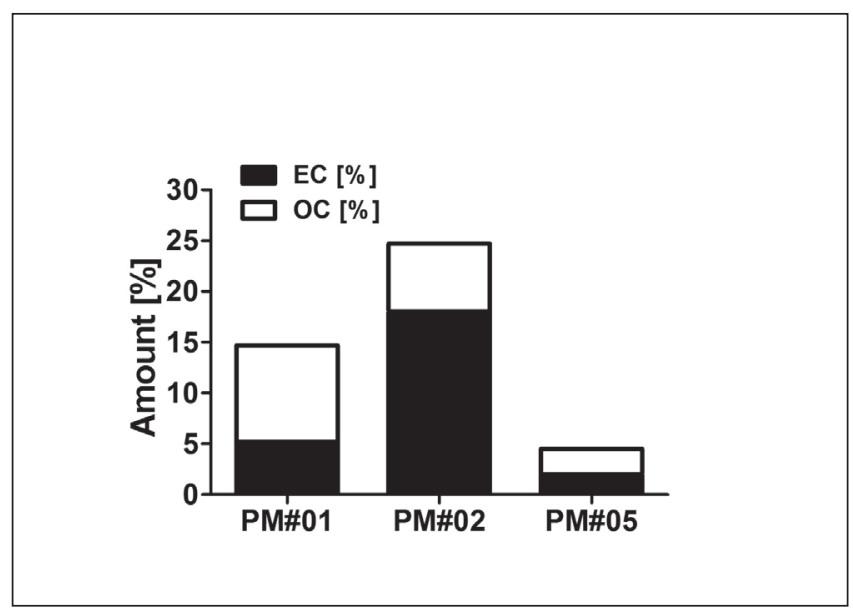

Fig. 19: Amount [\% dw] of elementary (EC) and organic carbon (OC) of PM\#01, PM\#02, and PM\#05 
Tab. 5: Overview of number weighted undersize values of non-fluorescent and fluorescent latex beads measured in the Mastersizer

Mean $\pm S D(n=3)$

\begin{tabular}{|c|c|c|c|c|c|c|}
\hline & \multicolumn{3}{|c|}{ Medium } & \multicolumn{3}{|c|}{ Water } \\
\hline & \multicolumn{6}{|c|}{ Non-fluorescent Latex Beads } \\
\hline & $50 \mathrm{~nm}$ & $200 \mathrm{~nm}$ & $1,000 \mathrm{~nm}$ & $50 \mathrm{~nm}$ & $200 \mathrm{~nm}$ & $1,000 \mathrm{~nm}$ \\
\hline $\mathrm{d}(0.1)[\mathrm{nm}]$ & $31.0 \pm 0.0$ & $119.67 \pm 2.0$ & $454.66 \pm 0.5$ & $54.33 \pm 39.5$ & $117 \pm 0.0$ & $445 \pm 1.0$ \\
\hline $\mathrm{d}(0.5)[\mathrm{nm}]$ & $54.66 \pm 0.5$ & $155.66 \pm 5.0$ & $643.33 \pm 0.5$ & $77.33 \pm 38.6$ & $148 \pm 1.0$ & $624 \pm 0.0$ \\
\hline \multirow[t]{2}{*}{$\mathrm{d}(0.9)[\mathrm{nm}]$} & $86.66 \pm 0.5$ & $236.00 \pm 8.5$ & $947.00 \pm 1.0$ & $116.0 \pm 48.5$ & $221.33 \pm 0.5$ & $914.0 \pm 0.0$ \\
\hline & \multicolumn{6}{|c|}{ Fluorescent Latex Beads } \\
\hline $\mathrm{d}(0.1)[\mathrm{nm}]$ & $31.00 \pm 0.0$ & $86.66 \pm 41.2$ & $412.33 \pm 16.9$ & $50.66 \pm 29.0$ & $108.33 \pm 1.5$ & $480.00 \pm 0.0$ \\
\hline $\mathrm{d}(0.5)[\mathrm{nm}]$ & $54.33 \pm 0.5$ & $134.00 \pm 13.8$ & $592.66 \pm 26.6$ & $80.33 \pm 24.3$ & $140.66 \pm 2.0$ & $670.66 \pm 0.5$ \\
\hline $\mathrm{d}(0.9)[\mathrm{nm}]$ & $86.00 \pm 0.0$ & $212.66 \pm 4.1$ & $966.00 \pm 20.5$ & $119.66 \pm 30.2$ & $214.33 \pm 4.1$ & $975.00 \pm 0.0$ \\
\hline
\end{tabular}

Tab. 6: Overview of number weighted undersize values of $1 \mathrm{mg} / \mathrm{ml}$ Aerosil200, DQ25, PM\#01, PM\#02 and PM\#05 in cell culture medium and water measured in the Mastersizer

Mean \pm SD $(n=3)$

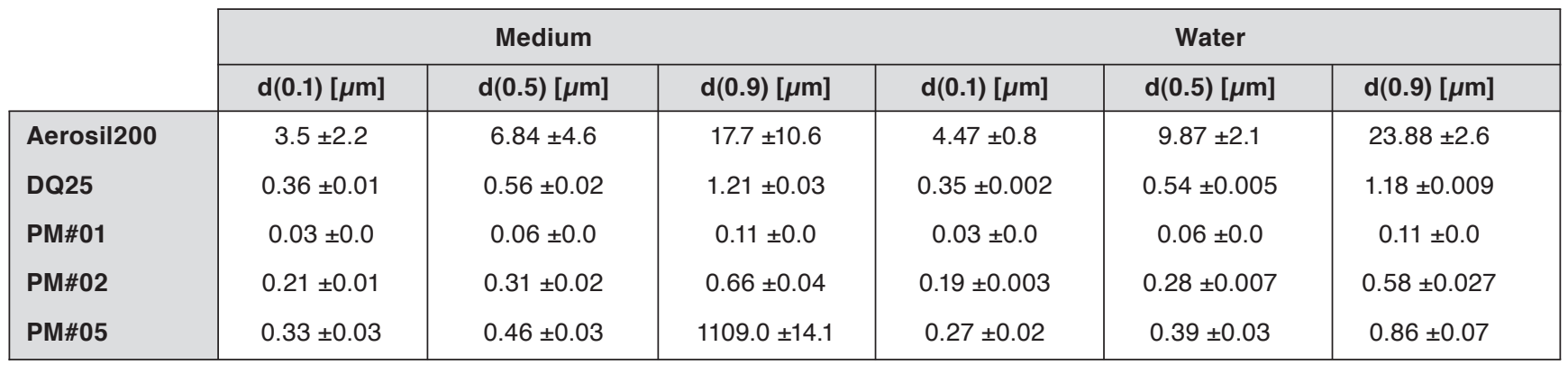

sion in cell culture media, again supporting earlier findings of aggregation of Aerosil200 (Fig. 18). Similarly, no differences between media and water were observed for DQ25 confirming the findings of lack of aggregation of DQ25 suspensions determined with other methods. Confirming the trend of the Mastersizer evaluations above, Nanosight measurement of PM\#01 showed smaller particles (cell culture medium: $81 \pm 43 \mathrm{~nm}$, water: $76 \pm 24 \mathrm{~nm}$ ) than PM\#02 (cell culture medium: $315 \pm 40 \mathrm{~nm}$, water: $256 \pm 27 \mathrm{~nm}$ ). PM\#05 was not evaluated.

\section{Centrifugation}

Centrifuged samples were measured with Zetasizer and Mastersizer and are displayed as intensity and volume weighted size distributions, respectively (Fig. S6, S7). Generally the Zetasizer determinations suggested that centrifugation pellets contained larger particles than the supernatant, irrespective of the particle type (Aerosil200, DQ25, or PM) and medium. Similarly, the Mastersizer determinations demonstrated that, with the exception of Aerosil200, all particle types appeared larger (more aggregates) in cell culture media than in water and larger particles were found in the pellet than in the supernatant.

\section{PAH analysis in PM samples}

Quantitative analysis of the three PM samples revealed the highest PAH amounts in samples from incomplete combustion processes, i.e., PM\#01 with $84.7 \mathrm{mg} / \mathrm{kg}$ and PM\#02 with $\sim 648 \mathrm{mg} / \mathrm{kg}$ (Tab. 7). PM\#05, resulting from a complete combustion process, had only $10.6 \mathrm{mg} / \mathrm{kg}$.

\section{Salt, elementary $(E C)$ and organic carbon $(O C)$}

analysis of PM samples

Correspondent to the PAH analysis, the highest total carbon amount was measured for PM\#02 (EC: 18\% dw, OC: $6.7 \% \mathrm{dw}$ ), followed by PM\#01 (EC: $5.2 \% \mathrm{dw}, \mathrm{OC}: 9.5 \% \mathrm{dw}$ ) and PM\#05 (EC: $2 \% \mathrm{dw}$, OC: $2.5 \% \mathrm{dw}$ ) (Fig. 19). PM\#01 presented the highest salt content (Tab. 8), albeit a direct comparison is misleading as the type and characteristics of the wood fuels used for generating the three different PM samples were different. Indeed, Ragland and coworkers demonstrated that salt content in wood fuels largely depends on wood type as well as on handling conditions (contamination) during wood harvesting (Ragland et al., 1991). 
Tab. 7: Amounts of the 16 US EPA PAHs detected in the PM samples

\begin{tabular}{|l|r|r|r|}
\hline PAH [mg/kg] & PM\#01 & PM\#02 & PM\#05 \\
\hline Naphthalin & 0.7 & $<4$ & $<0.4$ \\
Acenaphthylene & $<0.7$ & 6 & $<0.4$ \\
Acenaphthene & $<0.7$ & $<4$ & $<0.4$ \\
Fluorene & $<0.7$ & $<4$ & $<0.4$ \\
Phenanthrene & 2.5 & 75 & 0.8 \\
Anthracene & $<0.7$ & 15 & $<0.4$ \\
Fluoranthene & 2.9 & 142 & 1.3 \\
Pyrene & 2.8 & 137 & 1.2 \\
Benzo(a)anthracene & 2 & 42 & 0.7 \\
Chrysene & 2.2 & 55 & 1 \\
Benzo[b]fluoranthene & 8.9 & 63 & 1.8 \\
Benzo[k]fluoranthene & 2.6 & 20 & 0.5 \\
Benzo[a]pyrene & 7.8 & 26 & 0.7 \\
Indeno[1,2,3-c,d]pyrene & 25.4 & 35 & 1.4 \\
Dibenzo[a,h]anthracene & 2.8 & 4 & $<0.4$ \\
Benzo[g,h,i]perylene & 24.1 & 28 & 1.2 \\
\hline Sum & $\mathbf{8 4 . 7}$ & $\mathbf{6 4 8}$ & $\mathbf{1 0 . 6}$ \\
\hline
\end{tabular}

Tab. 8: Amounts of ions detected in the PM samples

\begin{tabular}{|l|r|c|c|}
\hline lons [mg/kg] & PM\#01 & PM\#02 & PM\#05 \\
\hline Fluoride & 806 & 0.96 & 0.004 \\
Chloride & 47581 & 42.4 & 55.2 \\
Nitrite & 6573 & 0.004 & 27.2 \\
Nitrate & 6008 & 1.080 & 0.004 \\
Phosphate & 806 & 0.004 & 4.8 \\
Sulfate & 233065 & 151.6 & 287.2 \\
\hline
\end{tabular}

Cytotoxicity assay

None of the three PM samples tested presented with overt cytotoxicity upon submersion exposure of A-549 cells, irrespective of the concentrations employed (Fig. 20).

\section{PAH CALUX ${ }^{\circledR}$ assay}

As expected from the PAH analyses (Tab. 7), PM\#01 and \#02 from incomplete combustions also induced the highest luciferase activity, irrespective of the application form (extract or as particles) in the transactivation assay, when compared to PM\#05 with a low PAH content and correspondingly low PAH CALUX ${ }^{\circledR}$ activity (Fig. 21). However, contrary to expectations, the total PAH content did not linearly correspond to a comparably high PAH CALUX ${ }^{\circledR}$ response. Indeed, PM\#02 contained approximately 61-fold more PAH than PM\#05, but resulted in only a 9-fold higher response in the PAH CALUX ${ }^{\circledR}$ when using PM-extracts, whereas a 252-fold higher response

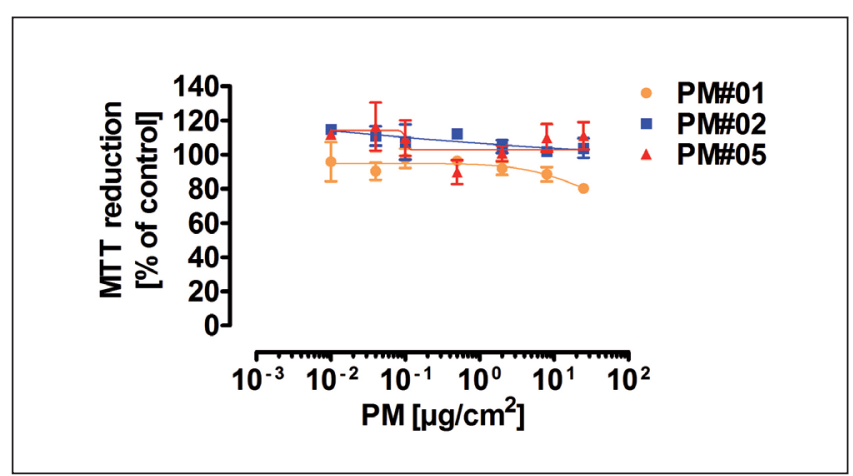

Fig. 20: Measurement of cytotoxicity via MTT reduction (in \% of control) upon $48 \mathrm{~h}$ exposure of A-549 cells to PM\#01, $\mathrm{PM \# 02}$ and PM\#05

Mean \pm SEM is shown $(n=3)$.

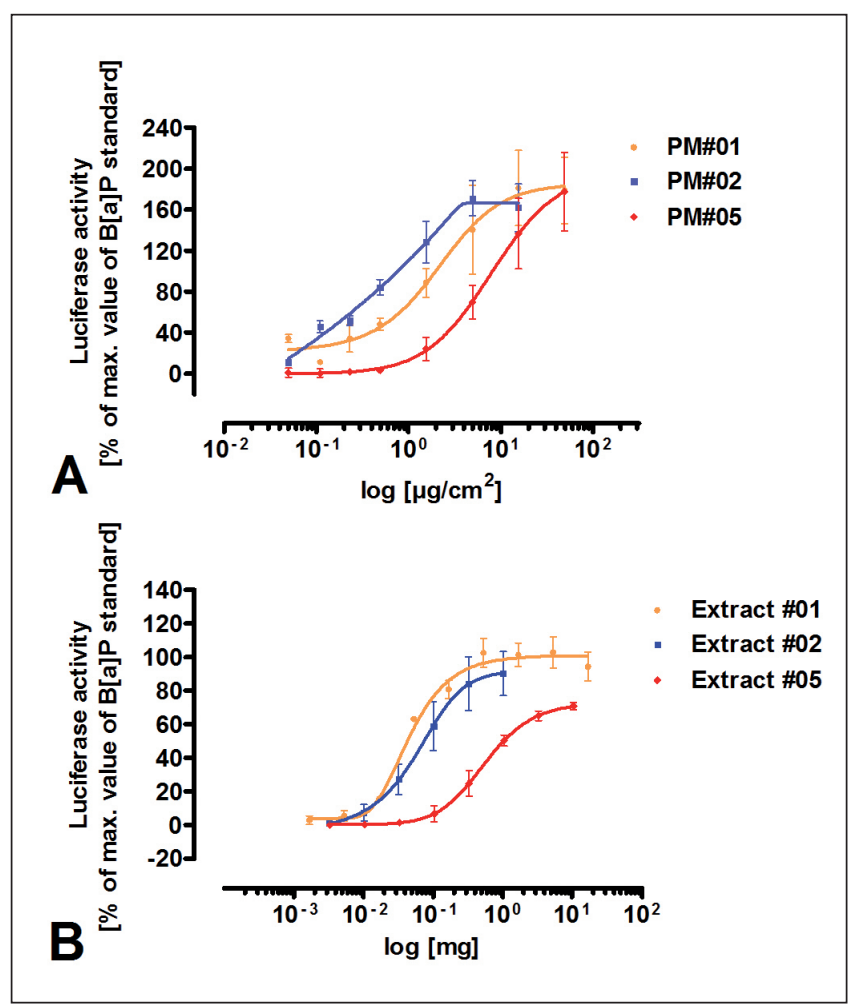

Fig. 21: Luciferase activity of PM\#01, PM\#02 and PM\#05 (A) as well as their respective extracts \#01, \#02, \#05 (B) in the PAH CALUX® assay.

Mean \pm SEM is shown $(n=3)$.

was observed when PM particles were tested (Tab. 9). Similarly, PM\#01 had an approximately 8-fold lower PAH content than PM\#02. However, contrary to expectations, the PAH CALUX $^{\circledR}$ response for PM\#01 extract was approximately 1.3-fold higher than for PM\#02, whereas the response to PM particles was comparable. The latter strongly suggests that total PAH is a poor reflection of real PAH bioavailability in a cell system. Indeed the data suggest that PAH bioavailability from 
Tab. 9: Comparison of total PAH amount and $\mathrm{EC}_{50}$ values of PM\#01, PM\#02 and PM\#05

\begin{tabular}{|c|c|c|c|c|c|c|}
\hline Sample & $\begin{array}{l}\text { PAH total } \\
(\mathrm{mg} / \mathrm{kg})\end{array}$ & $\begin{array}{c}\text { Factor PAH } \\
\text { total (compared } \\
\text { to PM\#02) }\end{array}$ & $\begin{array}{l}\mathrm{EC}_{50} \mathrm{PM} \\
\left(\mu \mathrm{g} / \mathrm{cm}^{2}\right)\end{array}$ & $\begin{array}{c}\text { Factor } \mathrm{EC}_{50} \\
\text { (compared to } \\
\text { PM\#02) }\end{array}$ & $\begin{array}{l}\mathrm{EC}_{50} \\
\text { Extracts } \\
(\mathrm{mg})\end{array}$ & $\begin{array}{c}\text { Factor } \mathrm{EC}_{50} \\
\text { (compared to } \\
\text { Extract PM\#02) }\end{array}$ \\
\hline \#01 & 84.7 & 7.7 & 1.947 & 64.9 & 0.0457 & 0.74 \\
\hline$\# 02$ & 648 & 1 & 0.03 & 1 & 0.0614 & 1 \\
\hline \#05 & 10.6 & 61.1 & 7.562 & 252 & 0.55 & 8.96 \\
\hline
\end{tabular}

the PM is the determinant potentially driving the biological activity, thus indicating that the combination of PM particle type (soot, salt, etc.), size, and PAH generated during the combustion process are the critical factors for the potential biological activity (toxicity) of the PM.

\section{Discussion}

A careful particle characterization in suspension has become an important prerequisite for nanomaterial toxicity testing (Warheit, 2008). Characterization is sometimes hindered by typical characteristics of nanomaterials, such as aggregation. Engineered nanoparticles have the advantage of being homogenous with regard to particle size, form, and composition and thus are ideally suited for size measurement via multiple methods. In contrast to the latter, wood combustion particles represent very complex and inhomogeneous mixtures.

Due to their presumed adverse effects on human health, as suggested by epidemiological studies, these particles have received increasing regulatory attention in the past years. However, toxicity testing and corresponding extrapolation to the human for proper risk assessment and risk management has proven extremely difficult. Indeed, very few animal studies have been carried out, many of which appear irreproducible due to variable and poor characterization of wood fuel and experimental conditions used, while a number of in vitro studies are difficult to compare to the animal and human findings and vice versa. The major caveat in the latter studies, irrespective of their nature (in vivo, in vitro or epidemiologic), is a proper characterization of the PM employed (Iba et al., 2006), thus rendering a comparison of data nearly impossible and not allowing a proper interpretation of the biological effects observed.

Indeed, PM from wood combustion exhaust are extremely complex and variable with regard to their type (soot, salt, etc.), form, size, and toxic contaminant content, which are largely dependent on the wood fuel used for combustion as well as on the furnace type and combustion conditions employed. Consequently, this study focused on the characterization of three different PM samples, whereby engineered fluorescent and nonfluorescent polystyrene particles (latex beads) as well as defined size mixtures of quartz particles were employed as reference materials for different size measurement methods. Moreover, a chemical characterization, determination of cytotoxicity, and the potential biological response of PM adsorbed toxins was specifically carried out for the wood combustion PM, thus providing for a better insight as to which PM characteristics would be important for an improved interpretation of potential health effects of wood combustion PM.

In view of the fact that particles, once inhaled, are dissolved in the fluid lining of the airways, the question was raised whether or not the airway lining fluid would have any influence on the physical characteristics of particles, i.e., whether aggregates could be formed within the airway lining fluid (ALF), thus preventing deeper penetration of particles into the alveolar area and enhancing mucociliary clearance (Goerke, 1998). Indeed, Lankoff and coworkers showed that aggregated $\mathrm{Ag}$ and $\mathrm{TiO}_{2}$ nanoparticles had cytotoxic effects. However, these effects were more pronounced for the least aggregated particles. Moreover, aggregation was influenced by the type of suspension preparation. The effects also were influenced by the type of particle and cell type used (Lankoff et al., 2012). As it was impossible to obtain sufficient amounts of human ALF for thorough testing of the particle types chosen, a comparison was carried out between particle suspensions in water and submersion culture medium (with/without HEPES), the latter representing the typical medium used for in vitro submerse cultures, as well as approximating the physico-chemical characteristics of airway lining fluid (Goerke, 1998).

The comparison of size measurements of latex beads suspended in nanopure water and culture medium did not demonstrate the presence of large aggregates. Indeed, none of the particle or PM types demonstrated an increased trend to form large size aggregates in cell culture media, with the exception of Aerosil200, as also demonstrated by the various methodologies employed for particle characterization (Fig. 4, 5, 9, 10, 15, 16, 18, S4). The concurrent analysis of PM also did not demonstrate the occurrence of larger aggregates, thus supporting the initial hypothesis that particles would behave similarly in nanopure water and cell culture medium. Thus it may be hypothesized that particles or particle mixtures, e.g., PM, would have comparable suspension characteristics in cell culture media under submerse in vitro cell culture conditions as in the fluid film covering the epithelial lining of the lung and nasopharyngeal space.

The analysis of Aerosil200 demonstrated the presence of large aggregates in cell culture media by qualitative light microscopy (Fig. 4), as well as following Zetasizer analyses (Fig. 9B, 10B , S2D-F), irrespective of the evaluation format of the Zetasizer data employed. As the corresponding Mastersizer analyses did not demonstrate the presence of an increased number of Aerosil200 aggregates in cell culture medium (Fig. 
$16,15 \mathrm{~A}-\mathrm{B})$, it must be assumed that the number of aggregates formed was too low for detection or that the stability to form aggregates was limited in the cell culture medium suspensions or was more pronounced for the water suspensions in the sodium polyphosphate medium used for the Mastersizer analyses (Fig. 16B). The latter data confirmed the potential of Aerosil200 to form large aggregates in cell culture media, possibly as a result of particle protein interactions and other compounds present in the cell culture medium, as already reported earlier for gold nanoparticles by Dobrovolskaia et al. (2008, 2009). It is thus considered likely that Aerosil200 also would form aggregates in the fluid film covering the epithelial lining of the lung and nasopharyngeal space.

Thus the current data demonstrate that, beside other potential factors (medium characteristics, suspension preparation techniques), specifically the particle type, here Aerosil200, appeared to influence aggregation behavior of the suspensions (Fig. S2D-F). The latter findings are corroborated by Ji et al. (2010) who found that the concentration of BSA in the respective media was negatively correlated with $\mathrm{TiO}_{2}$ aggregate size, suggesting that BSA enhanced homogeneous suspensions of $\mathrm{TiO}_{2}$ particles. Proteins in the medium influence, for example, the particle size upon building a protein corona around it (Dobrovolskaia et al., 2009). Moreover, nanoparticle size and surface properties also determine the protein corona that is developed (Lundqvist et al., 2008). Despite the latter, it remains unexplained why of all particle types employed in this study only Aerosil200 formed visible aggregates in cell culture media, while more inhomogeneous and chemically diverse particle mixtures, e.g., the combustion particulate matter (PM\#01, PM\#02 and PM \#05), did not.

However, the data do demonstrate that particle size and aggregation characterization can be achieved even for complex mixtures with a small subset of methods. Indeed, as demonstrated in Table 4, SEM evaluation is clearly helpful in providing a first impression of the homogeneity of the PM samples of interest, while light microscopy provides a first indication of the potential of a PM for forming aggregates in suspensions. Zetasizer or Mastersizer analyses will provide acceptable to optimal determination of particle size, as well as proof of PM aggregates, provided the number of aggregates is sufficiently high to be detected with the Mastersizer.

Indeed, for example, an upper grain size of $\mathrm{d}_{97 \%}$ with $18 \mu \mathrm{m}$ and $\mathrm{d}_{50} \%$ with $4 \mu \mathrm{m}$ for DQ25 (Tab. 3) was provided by the manufacturer of DQ25. However, according to number weighted measurements with Zetasizer and Mastersizer, as well as Nanosight measurements, smaller sizes were detected. The Zetasizer measurements yielded sizes between 600 and 2,000 $\mathrm{nm}$, consistent with Mastersizer measurements with sizes in cell culture medium and water between 300 and 4,000 nm, whereas the Nanosight was able to detect the smaller size fractions between 250-300 nm (Fig. 16, 18).

Consequently, Nanosight determinations may be helpful for size characterization. With an upper range of $<1 \mu \mathrm{m}$ Nanosight, however, measurements may prove unreliable, especially when analyzing complex particle mixtures with highly variant particle sizes and forms, e.g., PM (Tab. 4).
Although the data generated suggest that Zetasizer analyses are optimal, even for complex and inhomogeneous PM, sedimentation of bigger particles in an inhomogeneous particle suspension may profoundly influence the result of dynamic light scattering measurements. The latter was especially apparent in the quartz samples and PM samples requiring shorter measurement times to avoid the potential influence of particle sedimentation. Indeed, additional centrifugation experiments (Fig. S6, S7) suggested that larger particles could influence measurements of inhomogeneous suspension, as larger particles were detected in the centrifugation pellet than in the corresponding supernatant.

In contrast, due to measurement in a steady liquid flow passing the laser beam, the problem of sedimentation is avoided in the Mastersizer measurements. However, as particle suspensions have to be diluted in a sodium polyphosphate solution, an additional factor influencing particle aggregation is introduced that may affect Mastersizer measurements. In the latter methodology, inherent specificities may explain part of the differences observed between Zetasizer and Mastersizer results. Although the Mastersizer measurements of nanometer size particle suspensions should be interpreted with caution, the Mastersizer provides a larger determinable size range than the Zetasizer or Nanosight (Tab. 4).

As shown in the detailed evaluation of the Zetasizer and Mastersizer data, a true effect should be concluded from the data only when all data formats (intensity, volume, or number weighted means) of the same methodology demonstrate significant differences (Fig. 9B, S2D-F) and when differences are large. The latter also suggest that "false positive" significant differences can be generated in these analyses when differences are small and when only one of the data formats shows a significant difference whereas the others do not (Fig. S2A-C, S3-S5).

Thus careful data interpretation of the particle suspension characteristics is key to understanding particle distribution within a cell culture medium and thus understanding the potential availability of individual particles to the exposed cells (Dobrovolskaia et al., 2008; Lundqvist et al., 2008), as well as the ensuing biological effects determined (Baulig et al., 2004; Kocbach et al., 2008; Schwarze et al., 2007; Wick et al., 2007). Taken together, the current data suggest that i) both the Zetasizer and Mastersizer analyses will provide reliable size and aggregation characterization, even for inhomogeneous samples with unknown size distribution, e.g., PM from wood combustion; and ii) despite the possible advantages of Mastersizer measurements, one single method will not suffice for particle characterization or size determination, as limitations of the individual methods (Tab. 4), as well as individual sample preparation, will strongly influence the outcome of the analysis and the cellular responses observed (Lankoff et al., 2012). Consequently, a minimum of two methods not employing the same physical principle for aggregate or size determination will be required for a reliable particle characterization.

Beyond size and aggregation potential characterization, the type (nature) of the particles is of utmost importance. While latex beads and quartz particles are homogenous with regard to their composition, this is not the case for PM. Indeed, the three 
PM samples analyzed differ dramatically with regard to their content of salts, soot, and toxic contaminants, e.g., PAH (Tab. 7). The latter is a result of the type and form of wood employed, the water, bark, and salt content of the wood (Ragland et al., 1991), as well as of the type of furnace and combustion conditions chosen. As suggested in Figure 3, an incomplete combustion process is more likely to produce an inhomogeneous PM, e.g., PM\#01 and \#02, than a complete combustion (PM\#05) with a predominant proportion of smaller size particles. However, under medium suspension conditions PM\#05 with its smaller particles is more prone to produce an increased number of larger size aggregates (Fig. 17C, 15D). Obviously, the latter would most likely be an indication of whether these PM can reach the deep alveolar space or whether a major proportion of the material is aggregated in the ALF and then excreted via mucociliary clearance (Goerke, 1998). Thus the physico-chemical behavior within respiratory system, the type (primary salt or soot particles) and the toxin content of the PM will govern the biological response.

Upon testing the three different PM, none of the PM demonstrated an overt cytotoxicity, thereby corroborating earlier findings by Danielsen and coworkers (2011) who found no cytotoxicity with the LDH assay in A-549 and THP-1 cells after exposure to PM of different sample sites and of different combustion conditions. However, when a more refined biological endpoint was employed, i.e., the determination of whether any of these PM could interact with the Ah-receptor, using the PAH CALUX $^{\circledR}$ Ah-receptor transactivation assay, a dramatic difference in response was observed (Fig. 21). Organic extracts of the tested PM should contain nearly all of the PAHs present. Dissolved in DMSO, all of the PAH originating from the PM samples should be biologically available for binding to the Ahreceptor in vitro. Concomitant with the latter, the higher the PAH content of the PM samples, the stronger the response in the PAH CALUX ${ }^{\circledR}$ Ah-receptor transactivation assay.

However, as demonstrated in Figure 21 and Table 9, the total amount of PAH detected in the PM samples did not correspond to the response observed in vitro. Indeed, although PM\#02 contained approximately 8-times more PAH than PM\#01, the extracts of PM\#01 and \#02 were nearly equipotent (0.7-fold) in the PAH CALUX ${ }^{\circledR}$ assay. Moreover, PM\#02 contained approximately 61-times more PAH than PM\#05, but the extracts of PM\#02 were only 9-fold more potent than those of PM\#05. Obviously the latter interpretation is based on the incorrect assumption that all PAH are equipotent in their binding affinity and capacity to the Ah-receptor. Indeed, it is the individual PAH congener and its respective concentration that drives the PAH CALUX $^{\circledR}$ assay response.

As the total concentration of PAH with known high affinity for the Ah-receptor and transactivation capability is much higher in PM\#02 than in PM\#01, it is assumed that the high concentrations of the other PAH present (phenanthrene, anthracene, fluoranthene, pyrene) may have weakly or nonspecifically bound to the Ah-receptor and thus competed with the specific binding of the Ah-receptor interactants in the assay. Moreover, the chemical PAH analysis only encompassed the 16 US EPA PAHs. Of these, only a fraction are capable of interacting (acti- vating) with the Ah-receptor in the PAH CALUX ${ }^{\circledR}$ assay. However, there are many more PAHs known with activity towards the Ah receptor, that are not detected via the 16 US EPA PAH analysis. Consequently, differences, or the lack thereof, between extracts of PM\#01 and \#02 also may have resulted from Ah-receptor active PAHs in the extracts not detected with the routine 16 US EPA PAH analysis.

When comparing the response of PM as suspensions in the PAH CALUX ${ }^{\circledR}$ assay, the potency of the mixtures were PM\#02 $>$ PM\#01 >> PM\#05 (Fig. 21A, Tab.9). However, contrary to the finding with the extracts, PM\#01 was approximately 65-times less potent than PM\#02, suggesting that not all relevant PAHs for Ah-receptor interaction were biologically available from the PM\#01 particle suspension. The same holds true for the comparison of particle suspensions and extracts of PM\#02 and PM\#05, where the particle suspension of PM\#05 was 252-fold less potent than an equal amount of PM\#02, despite the fact that PM\#02 only had a 68-fold higher PAH content.

The above findings, albeit preliminary and certainly requiring more in-depth investigation, suggest that the bioavailability of toxins (toxicokinetic component), in this case PAH, is governed by the physico-chemical composition of the PM and that the latter strongly influences the biological response (toxicodynamic component). More importantly, these data strongly suggest that the mere chemical analyses of PAH in a PM sample, as is current practice for the risk assessment of PM (Mumtaz and George, 1995), will not suffice to predict potential adverse effects.

\section{Supplementary data}

The supplementary data file at www.altex-edition.org displays the results of all conducted measurements not shown in this manuscript. Beside the $1 \mathrm{mg} / \mathrm{ml}$ concentration measurements presented in this paper, two further concentrations $(0.5$ and $2 \mathrm{mg} / \mathrm{ml})$ were measured for PM and quartz samples. Moreover, all result transformations of Zetasizer and Mastersizer measurements are shown.

\section{References}

Baulig, A., Poirault, J. J., Ausset, P., et al. (2004). Physicochemical characteristics and biological activities of seasonal atmospheric particulate matter sampling in two locations of Paris. Environ. Sci. Technol. 38, 5985-5992.

Berg, J. M., Romoser, A., Banerjee, N., et al. (2009). The relationship between $\mathrm{pH}$ and zeta potential of $\sim 30 \mathrm{~nm}$ metal oxide nanoparticle suspensions relevant to in vitro toxicological evaluations. Nanotoxicology 3, 276-283.

Billet, S., Abbas, I., Le Goff, J., et al. (2008). Genotoxic potential of Polycyclic Aromatic Hydrocarbons-coated onto airborne Particulate Matter (PM 2.5) in human lung epithelial A549 cells. Cancer Lett 270, 144-155.

Chylek, P. (1986). Absorption and scattering of light by small particles. By C. F. Bohren and D. R. Huffman. Appl. Opt. 25, 3166 .

Danielsen, P. H., Moller, P., Jensen, K. A., et al. (2011). Oxidative stress, DNA damage, and inflammation induced by am- 
bient air and wood smoke particulate matter in human A549 and THP-1 cell lines. Chem. Res. Toxicol. 24, 168-184.

Dobrovolskaia, M. A., Aggarwal, P., Hall, J. B., et al. (2008). Preclinical studies to understand nanoparticle interaction with the immune system and its potential effects on nanoparticle biodistribution. Mol. Pharm. 5, 487-495.

Dobrovolskaia, M. A., Patri, A. K., Zheng, J., et al. (2009). Interaction of colloidal gold nanoparticles with human blood: effects on particle size and analysis of plasma protein binding profiles. Nanomedicine 5, 106-117.

European Union (2008). Directive 2008/50/EC of the European Parliament and of the Council of 21 May 2008 on ambient air quality and cleaner air for Europe. Official Journal of the European Union L 152, 1-44.

Goerke, J. (1998). Pulmonary surfactant: functions and molecular composition. Biochim. Biophys. Acta 1408, 79-89.

Gualtieri, M., Mantecca, P., Cetta, F., et al. (2008). Organic compounds in tire particle induce reactive oxygen species and heat-shock proteins in the human alveolar cell line A549. Environ. Int. 34, 437-442.

Iba, M. M., Fung, J., Chung, L., et al. (2006). Differential inducibility of rat pulmonary CYP1A1 by cigarette smoke and wood smoke. Mutat. Res. 606, 1-11.

Ji, Z., Jin, X., George, S., et al. (2010). Dispersion and stability optimization of $\mathrm{TiO}_{2}$ nanoparticles in cell culture media. Environ. Sci. Technol. 44, 7309-7314.

Jones, C. F. and Grainger, D. W. (2009). In vitro assessments of nanomaterial toxicity. Adv. Drug Deliv. Rev. 61, 438-456.

Kocbach, A., Herseth, J. I., Lag, M., et al. (2008). Particles from wood smoke and traffic induce differential pro-inflammatory response patterns in co-cultures. Toxicol. Appl. Pharmacol. 232, 317-326.

Lankoff, A., Sandberg, W. J., Wegierek-Ciuk, A., et al. (2012). The effect of agglomeration state of silver and titanium dioxide nanoparticles on cellular response of HepG2, A549 and THP-1 cells. Toxicol. Lett. 208, 197-213.

Lundqvist, M., Stigler, J., Elia, G., et al. (2008). Nanoparticle size and surface properties determine the protein corona with possible implications for biological impacts. Proc. Natl. Acad. Sci. USA 105, 14265-14270.

Mumtaz, M. and George, J. (1995). Toxicological profile for polycyclic aromatic hydrocarbons. Agency for Toxic Substances and Disease Registry. http://www.atsdr.cdc.gov/ toxprofiles/tp69.pdf

Naeher, H., Smith, K. R., Brauer, M., et al. (2005). Critical review of the health effects of woodsmoke. http://ehs. sph.berkeley.edu/krsmith/publications/2005\%20pubs/ HC\% 20woodsmoke\% 20report\%20Mar\%2031\%2005\% 20\%28rev\%29.pdf

Naeher, L. P., Brauer, M., Lipsett, M., et al. (2007). Woodsmoke health effects: a review. Inhal. Toxicol. 19, 67-106.

Nora, C., Mabic, S., and Darbouret, D. (2002). A theoretical approach to measuring $\mathrm{pH}$ and conductivity in high-purity water. Ultrapure Water 10, 56-61.

Pope, C. A., $3^{\text {rd }}$, Burnett, R. T., Thun, M. J., et al. (2002). Lung cancer, cardiopulmonary mortality, and long-term exposure to fine particulate air pollution. JAMA 287, 1132-1141.

Pope, C. A., $3^{\text {rd }}$ and Dockery, D. W. (2006). Health effects of fine particulate air pollution: lines that connect. J. Air Waste Manag. Assoc. 56, 709-742.

Quaak, P., Knoef, H., and Stassen, H. (1999). Energy from biomass - A review of combustion and gasification technologies. World Bank Technical Paper No. 422.

Ragland, K. W., Aerts, D. J., and Baker, A. J. (1991). Properties of wood for combustion analysis. Bioresource Technology 37, 161-168.

Schwarze, P. E., Ovrevik, J., Hetland, R. B., et al. (2007). Importance of size and composition of particles for effects on cells in vitro. Inhal. Toxicol. 19, Suppl. 1, 17-22.

Taurozzi, J. S., Hackley, V. A., and Wiesner, M. R. (2010). Ultrasonic dispersion of nanoparticles for environmental, health and safety assessment - issues and recommendations. Nanotoxicology 5, 711-729.

Teeguarden, J. G., Hinderliter, P. M., Orr, G., et al. (2007). Particokinetics in vitro: dosimetry considerations for in vitro nanoparticle toxicity assessments. Toxicol. Sci.95, 300-312.

Warheit, D. B. (2008). How meaningful are the results of nanotoxicity studies in the absence of adequate material characterization? Toxicol. Sci. 101, 183-185.

Wick, P., Manser, P., Limbach, L. K., et al. (2007). The degree and kind of agglomeration affect carbon nanotube cytotoxicity. Toxicol. Lett. 168, 121-131.

\section{Acknowledgements}

We would like to acknowledge the financial support from the German Federal Ministry of Environment. The PM samples were kindly provided by the "Deutsches Biomasse Forschungszentrum Leipzig" and the "Technisches Förderzentrum Straubing". Furthermore, we would like to thank Professor Stefan Mecking, University of Konstanz, Dr Peter Wick, EMPA St. Gallen, and Dr Adrian Gilli, ETH Zürich, for cooperation with regard to particle characterization as well as Dr Joachim Hentschel and Professor Kurt Mendgen for technical support with SEM. Special thanks to Alexandra Heussner, Dr Jürgen Lühmann, Stefanie Wirth, Xenia Mäder, Dr Tina Turnherr, and Lars Bolk for technical assistance.

\section{Correspondence to}

Daniel Dietrich, $\mathrm{PhD}$

Human and Environmental Toxicology

Department of Biology

University of Konstanz

P.O. Box X-918

78457 Konstanz

Germany

Fax: +497531883170

Phone: +49 7531883518

e-mail: Daniel.Dietrich@uni-konstanz.de 